\title{
Community-Driven Code Comparisons for Three-Dimensional Dynamic Modeling of Sequences of Earthquakes and Aseismic Slip (SEAS)
}

\author{
Junle Jiang ${ }^{1}$, Brittany A. Erickson ${ }^{2}$, Valère R. Lambert ${ }^{3}$, \\ Jean-Paul Ampuero ${ }^{4}$, Ryosuke Ando ${ }^{5}$, Sylvain D. Barbot ${ }^{6}$, Camilla Cattania ${ }^{7}$, \\ Luca Dal Zilio $^{8,9}$, Benchun Duan ${ }^{10}$, Eric M. Dunham ${ }^{11}$, Alice-Agnes Gabriel ${ }^{12,13}$, \\ Nadia Lapusta ${ }^{8}$, Duo Li ${ }^{12}$, Meng Li ${ }^{14}$, Dunyu Liu ${ }^{15}$, Yajing Liu ${ }^{16}$, \\ So Ozawa ${ }^{5}$, Casper Pranger ${ }^{9,12}$, Ylona van Dinther ${ }^{9,14}$ \\ ${ }^{1}$ School of Geosciences, University of Oklahoma, Norman, OK, USA \\ ${ }^{2}$ Department of Computer and Information Science, University of Oregon, Eugene, OR, USA \\ ${ }^{3}$ Department of Earth and Planetary Sciences, University of California, Santa Cruz, CA, USA \\ ${ }^{4}$ Université Côte d'Azur, IRD, CNRS, Observatoire de la Côte d'Azur, Géoazur, France \\ ${ }^{5}$ Department of Earth and Planetary Science, University of Tokyo, Japan \\ ${ }^{6}$ Department of Earth Sciences, University of Southern California, Los Angeles, CA, USA \\ ${ }^{7}$ Department of Earth, Atmospheric, and Planetary Sciences, Massachusetts Institute of Technology, Cambridge, MA, USA \\ ${ }^{8}$ Seismological Laboratory and Department of Mechanical and Civil Engineering, \\ California Institute of Technology, Pasadena, CA, USA \\ ${ }^{9}$ Institute of Geophysics, Department of Earth Sciences, ETH Zurich, Zurich, Switzerland \\ ${ }^{10}$ Department of Geology and Geophysics, Texas A\&M University, College Station, TX, USA \\ ${ }^{11}$ Department of Geophysics, Stanford University, Stanford, CA, USA \\ ${ }^{12}$ Department of Earth and Environmental Sciences, Ludwig-Maximilians-Universität München, Munich, Germany \\ ${ }^{13}$ Institute of Geophysics and Planetary Physics, Scripps Institution of Oceanography, \\ University of California, San Diego, CA, USA \\ ${ }^{14}$ Department of Earth Sciences, Utrecht University, Utrecht, Netherlands \\ ${ }^{15}$ Institute for Geophysics, University of Texas at Austin, TX, USA \\ ${ }^{16}$ Department of Earth and Planetary Sciences, McGill University, Montréal, QC, Canada
}

\section{Key Points:}

- We pursue community efforts to develop code verification benchmarks for three-dimensional earthquake rupture and crustal faulting problems

- We assess the agreement and discrepancies of seismic and aseismic fault behavior among simulations based on different numerical methods

- Our comparisons lend confidence to numerical codes and reveal sensitivities of model observables to major computational and physical factors 


\begin{abstract}
Dynamic modeling of sequences of earthquakes and aseismic slip (SEAS) provides a self-consistent, physics-based framework to connect, interpret, and predict diverse geophysical observations across spatial and temporal scales. Amid growing applications of SEAS models, numerical code verification is essential to ensure reliable simulation results but is often infeasible due to the lack of analytical solutions. Here, we develop two benchmarks for three-dimensional (3D) SEAS problems to compare and verify numerical codes based on boundary-element, finite-element, and finite-difference methods, in a community initiative. Our benchmarks consider a planar vertical strike-slip fault obeying a rate- and state-dependent friction law, in a 3D homogeneous, linear elastic whole-space or half-space, where spontaneous earthquakes and slow slip arise due to tectonic-like loading. We use a suite of quasi-dynamic simulations from 10 modeling groups to assess the agreement during all phases of multiple seismic cycles. We find excellent quantitative agreement among simulated outputs for sufficiently large model domains and small grid spacings. However, discrepancies in rupture fronts of the initial event are influenced by the free surface and various computational factors. The recurrence intervals and nucleation phase of later earthquakes are particularly sensitive to numerical resolution and domain-size-dependent loading. Despite such variability, key properties of individual earthquakes, including rupture style, duration, total slip, peak slip rate, and stress drop, are comparable among even marginally resolved simulations. Our benchmark efforts offer a community-based example to improve numerical simulations and reveal sensitivities of model observables, which are important for advancing SEAS models to better understand earthquake system dynamics.
\end{abstract}

\title{
Plain Language Summary
}

Earthquakes and fault zone processes occur over time scales ranging from milliseconds to millennia and longer. Computational models are increasingly used to simulate sequences of earthquakes and aseismic slip (SEAS). These simulations can be connected to diverse geophysical observations, offering insights into earthquake system dynamics. To improve these simulations, we pursue community efforts to design benchmarks for 3D SEAS problems. We involve earthquake researchers around the globe to compare simulation results using different numerical codes. We identify major factors that contribute to the discrepancies among simulations. For example, the spatial dimension and resolution of the computational model can affect how earthquakes start and grow, as well as how frequently they recur. Code comparisons are more challenging when we consider the Earth's surface in the simulations. Fortunately, we find that several key characteristics of earthquakes are accurately reproduced in simulations, such as the duration, total movement, maximum speed, and stress change on the fault, even when model resolutions are not ideal. These exercises are important for promoting a new generation of advanced models for earthquakes. Understanding the sensitivity of simulation 
outputs will help test models against real-world observations. Our community efforts can serve as a useful example to other geoscience communities.

\section{Introduction}

Physics-based computational models of dynamic processes in the Earth are increasingly used to understand and predict observations from the lab and field across spatial and temporal scales, addressing fundamental questions in various branches of solid Earth research. In earthquake science, models of earthquake source processes are aimed at capturing dynamic earthquake ruptures from seconds to minutes and slow slip processes subject to short-term anthropogenic or environmental forcing, or tectonic loading over timescales of years and longer. For individual earthquakes, dynamic rupture simulations have emerged as powerful tools to reveal the influence of fault structure, geometry, constitutive laws, and prestress on earthquake rupture propagation and associated ground motion (e.g., Andrews, 1976a,b; Ben-Zion, 2001; Bhat et al., 2007; Bizzarri and Cocco, 2003, 2006; Day, 1982; Das and Aki, 1977; Duan and Day, 2008; Dunham et al., 2011a,b; Gabriel et al., 2012; Harris et al., 1991, 2021; Kozdon and Dunham, 2013; Lozos et al., 2011; Ma and Beroza, 2008; Madariaga et al., 1998; Mikumo and Miyatake, 1978, 1993; Nielsen et al., 2000; Olsen et al., 1997; Ripperger et al., 2007; Shi and Day, 2013; Tinti et al., 2021; Wollherr et al., 2019; Xu et al., 2015). These simulations are limited to single-event scenarios and subject to imposed artificial prestress conditions and ad hoc nucleation procedures. For larger-scale fault network systems, earthquake simulators aim to produce complex spatiotemporal characteristics of seismicity over millennial time scales (Richards-Dinger and Dieterich, 2012; Robinson and Benites, 1995, 1996, 2001; Shaw et al., 2018; Tullis et al., 2012). The formidable computational demand inevitably requires simplification and approximation of some key physical features that could influence or dominate earthquake and fault interactions, such as seismic waves, slow slip, tectonic loading, and inelastic response.

To understand earthquake system dynamics, it has been widely recognized that we need models that simulate fault behavior over multiple seismic events and the intervening periods of aseismic deformation. To address this need, numerical simulations of Sequences of Earthquakes and Aseismic Slip (SEAS) are developed to consider all phases of earthquake faulting, from slow loading to earthquake nucleation, propagation and termination over time scales of milliseconds to millennia in a unified, self-consistent framework (Figure 1; Ben-Zion and Rice, 1995; Lapusta et al., 2000; Rice, 1993). While retaining computational rigor, SEAS models incorporate the structure, rock properties, friction, and rheology of a fault zone, and produce the pre-, inter-, and post-seismic slip and the resulting stress redistribution that ultimately lead to spontaneous earthquake nucleation and dynamic ruptures. SEAS models can include many physical processes relevant to long-term slip, such as evolving shear resistance of the fault zone affected by shear heating, fluid effects, and interseismic healing, wave-mediated inertial effects during dynamic rupture, folding, viscoelasticity, 
and fluid flow (e.g., Allison and Dunham, 2018; Barbot, 2018; Lambert and Barbot, 2016; Noda and Lapusta, 2010; Sathiakumar et al., 2020; Thomas et al., 2014; Zhu et al., 2020). This modeling framework can help determine and quantify which physical factors control diverse observables such as ground deformation and shaking, and the frequency, size, and rupture style of microseismicity and large earthquakes. SEAS models also bridge the domains of dynamic rupture simulations and earthquake simulators, providing physically justified approximations and self-consistent choices for initial conditions and earthquake nucleation procedures.

Developments in SEAS models over the past two decades have led to increased diversity and complexity of models and closer connections between simulations and observations from the lab and field. For example, numerical models have been combined with seismic and geodetic observations to study fault frictional properties (e.g., Barbot et al., 2009; Dublanchet et al., 2013; Floyd et al., 2016; Hori et al., 2004; Jiang and Fialko, 2016; Johnson et al., 2006; Mitsui and Iio, 2011; Tymofyeyeva et al., 2019), tremor and slow slip (e.g., Dal Zilio et al., 2020; Dublanchet, 2018; Hawthorne and Rubin, 2013; Luo and Ampuero, 2018; Mele Veedu and Barbot, 2016; Shibazaki and Iio, 2003; Wang and Barbot, 2020), foreshock and aftershock sequences (e.g., Cattania and Segall, 2021; Kaneko and Lapusta, 2008; Perfettini and Avouac, 2007; Noda et al., 2013), and characteristics of small and large earthquake ruptures (e.g., Barbot et al., 2012; Cattania and Segall, 2019; Chen and Lapusta, 2009; Jiang and Lapusta, 2016, 2017; Lambert and Lapusta, 2021). The framework of earthquake sequence modeling is also adopted in diverse settings, which include subduction zones (e.g., Hori et al., 2004; Liu and Rice, 2005, 2007; Li and Liu, 2016, 2017; Shi et al., 2020; Van Dinther et al., 2013), collision zones (e.g., Dal Zilio et al., 2018; Michel et al., 2017; Qiu et al., 2016), and induced seismicity phenomena (e.g., Dieterich et al., 2015; Kroll and Cochran, 2021; McClure and Horne, 2011), among many applications.

While researchers continue to build more advanced and detailed SEAS models, verification of different numerical codes is essential to ensure credible and reproducible results, and sustain scientific progress. In practice, analytical solutions are generally not available, even for simple SEAS problems, and convergence of simulations to a high-resolution reference case may not always detect systematic issues in complex numerical codes. An alternative means for verifying model results are comparisons of independent numerical codes from different research groups. As an example, the SCEC/USGS Spontaneous Rupture Code Verification Project pioneered the code comparison exercise and improved confidence in the outcomes of dynamic rupture simulations (Barall and Harris, 2015; Day et al., 2005; Harris et al., 2009, 2018).

Verification of SEAS models is confronted with distinct challenges, due to the wide range of spatial and temporal scales that characterize the earthquake source behavior and the diversity of numerical algorithms and codes. For example, codes based on the spectral boundary element method (SBEM) (Barbot, 2021; Lapusta and Rice, 2003; Lapusta and Liu, 2009) are highly efficient 
in solving for fully dynamic earthquake ruptures, albeit with relatively simple fault geometry and bulk. Codes based on the boundary element method (BEM) (e.g., Barbot, 2019; Kato, 2016; Liu, 2013; Luo et al., 2017; Nakata et al., 2012; Rice and Tse, 1986; Segall and Bradley, 2012; Tse and Rice, 1986) can efficiently simulate earthquake ruptures in problems with more complex fault geometry, often with the approximation of inertia (i.e., quasi-dynamic earthquakes). Codes based on the finite difference method (FDM) (e.g., Allison and Dunham, 2018; Erickson and Dunham, 2014; Erickson et al., 2017; Herrendörfer et al., 2018; Mckay et al., 2019; Pranger, 2020), finite element method (FEM) (e.g., Liu et al., 2020; Luo et al., 2020; Tal and Hager, 2018), and spectral element method (SEM) (e.g., Kaneko et al., 2011; Thakur et al., 2020) can flexibly incorporate geometrical and structural complexity in earthquake simulations, usually at a greater computational cost than BEM. For all these codes, common challenges lie in the interaction between the highly nonlinear nature of the SEAS problems and numerical round-off errors, which can lead to the divergence of model behaviors with increasing simulated time (Lambert and Lapusta, 2021). Simulation techniques are further complicated when additional physical factors, e.g., fault roughness, material heterogeneities, and bulk inelastic responses, are incorporated or approximated (e.g., Abdelmeguid et al., 2019; Dal Zilio et al., 2022; Romanet and Ozawa, 2021). However, considering such complexity may be crucial in our efforts to understand earthquakes and predict seismic hazards.

This study represents ongoing community efforts in the SEAS working group, supported by the Southern California Earthquake Center (SCEC) to perform code verification exercises for SEAS models. We reported the community initiative and results from our first two benchmarks, BP1-QD and BP2-QD, for two-dimensional (2D) SEAS problems in Erickson et al. (2020). We gather 11 independent modeling groups using different numerical codes to participate and compare 2D SEAS simulations. Through code comparisons, we identify how various computational factors, such as the numerical resolution, domain size, and boundary conditions, influence simulation results in 2D antiplane problems. Our exercises demonstrated excellent agreement in simulations with a sufficiently small grid spacing and large domain size, lending confidence to the participating numerical codes. We also found that artificial complexity in earthquake patterns can arise due to insufficient numerical resolution for key physical length scales, although ensemble-averaged measures, such as earthquake recurrence times, are more robust than observables from individual simulations, even at poor numerical resolutions.

As our community and code capabilities grow, we have made substantial progress in benchmark efforts for three-dimensional (3D) SEAS problems. Here, we present our recent development of two new 3D benchmarks, BP4 and BP5. The dramatically increased computational demand for 3D problems requires us to balance the simplicity and realism of the benchmark problems (Section 2). Although we present the complete benchmark descriptions that include both fully dynamic (FD; including inertia) and quasi-dynamic (QD; approximating inertia) formulations of earthquake ruptures, 
our code comparison results are limited to the quasi-dynamic problems. We examine choices of numerical implementations among the modeling groups to ensure consistent comparisons of a large set of 3D simulations (Section 3). We also design new strategies and metrics for code verification for complex 3D simulations that are often done at the upper limit of numerical resolutions (Section 4). In particular, we explore the sensitivity of diverse model outputs and observables to major computational and physical factors. Through these efforts, we aim to improve and promote a new generation of rigorous, robust numerical codes for SEAS problems, and to inform and interact with other communities that are tackling similar computational challenges in nonlinear, multiscale, multi-physics problems (e.g. Buiter et al., 2016; Matsui et al., 2016; Maxwell et al., 2014; Nearing et al., 2018).

\section{Community Benchmark Development}

\subsection{Strategy for Benchmark Design}

We follow the principle of starting simple and incrementally adding complexity in the design process of SEAS benchmarks. For 2D benchmark problems (BP1-QD and BP2-QD), a 1D fault in a 2D antiplane setting was considered to explore how the computational domain size and boundary conditions affect simulation results and how numerical resolution (grid spacing or cell size) influences earthquake patterns and statistics (Erickson et al., 2020). Overall, we aim to verify different numerical codes through a detailed comparison of simulated fault behavior over multiple time scales. These efforts require a better understanding of the dependence of fault slip history on fault properties, friction laws, initial conditions, model spin-up, and other factors.

Our findings and experience from 2D benchmark exercises prepare us for more complicated 3D benchmark problems. We need to design 3D benchmarks that are tractable for the widest suite of numerical codes and thereby maximize participation of modelers, especially considering the higher computational cost of 3D simulations and distinct capabilities of different codes in the community. For example, codes based on the spectral boundary element method, e.g., BICyclE (Lapusta and $\mathrm{Liu}, 2009$ ), are efficient in solving for quasi-dynamic or fully dynamic earthquake ruptures, but rely on periodic boundary conditions and free surface approximations. Methods based on the finite element method, e.g., EQsimu (Liu et al., 2020), can incorporate more complicated fault geometries and bulk, including a rigorous treatment of the free surface, but need to balance the domain size with a reasonable computational cost.

While we can in principle compare the full spectrum of fault behavior in SEAS models, the focus of our exercise here is on reproducing earthquake nucleation, rupture, and recurrence. With the computational cost in mind, we design benchmark problems where a direct comparison of individual earthquakes is feasible (hence a consistent nucleation location is desirable). We then assess the agreement of important model observables and their sensitivity to computational and 
physical factors. A better understanding of the roles of various inputs and outputs in SEAS models will guide us in developing more complicated benchmarks and validating SEAS models in future.

Since the participation of many modelers is essential to the success of the code verification exercise, we seek to build a consensus in the community at the outset of our benchmark design process. We conducted surveys among the interested modelers to decide on the most preferred benchmark problems. For instance, we have chosen to focus on quasi-dynamic problems for our initial 3D benchmarks, BP4 and BP5, given that many numerical codes cannot yet incorporate full inertial effects but adopt the radiation damping approximation (Rice, 1993). While we assess a myriad of simulation outputs and develop metrics for model comparisons, we are flexible about the submitted simulation data, given that sometimes substantial code development is needed. During the subsequent development following initial comparisons of benchmark BP4, we learned lessons about the computational cost and have accordingly revised the model parameters and output types for benchmark BP5, hence some minor differences exist between the two benchmarks.

\subsection{Benchmark Problem Setup}

We have developed two benchmarks, BP4 and BP5, for 3D SEAS simulations (Figure 2). Our first 3D benchmark problem, BP4, considers a 3D homogeneous, isotropic, linear elastic whole space in $\mathbb{R}^{3}$, defined by $\boldsymbol{x}=\left(x_{1}, x_{2}, x_{3}\right) \in(-\infty, \infty)^{3}$, where $x_{1}, x_{2}$, and $x_{3}$ refer to the coordinates in the fault-normal, along-strike, and along-dip directions, respectively. A vertical strike-slip fault is embedded at $x_{1}=0$. We use the notation "+" and "-" to refer to the side of the fault with $x_{1}$ positive and negative, respectively. We assume 3D motion, denoting components of the displacement vector $\boldsymbol{u}$ as $u_{i}=u_{i}(\boldsymbol{x}, t), i=1,2,3$, in the $i$-direction. The second 3D benchmark problem, BP5, involves a fault with half the vertical dimension in a 3D half-space, defined by $\boldsymbol{x}=\left(x_{1}, x_{2}, x_{3}\right) \in(-\infty, \infty) \times(-\infty, \infty) \times(0, \infty)$, with a free surface at $x_{3}=0$ and $x_{3}$ as positive downward. Several model parameters in BP5 are adjusted to allow for reduced computational demand compared with BP4.

Each benchmark problem branches into two versions, depending on the treatment of the inertial effect, i.e., quasi-dynamic (QD) or fully dynamic (FD) earthquake ruptures, which are assigned with different suffixes in benchmark names (e.g., BP4-QD or BP4-FD). Full descriptions of these benchmarks are available online on the SEAS code comparison platform (https://strike. scec.org/cvws/seas/) and also included as supplementary materials. We summarize below the governing equations, constitutive laws, and initial and interface conditions that are important for understanding SEAS simulations for both QD and FD problems, and related numerical resolution issues. For consistency and clarity, we have changed a few notations from the original benchmark descriptions. 
The 3D fault zone motion is governed by the momentum balance equation, or the equilibrium equation if inertia is neglected:

$$
\begin{aligned}
\rho \frac{\partial^{2} u}{\partial t^{2}} & =\nabla \cdot \sigma & & \text { for FD problems } \\
0 & =\nabla \cdot \sigma & & \text { for QD problems }
\end{aligned}
$$

where $\boldsymbol{u}$ is the displacement vector, $\boldsymbol{\sigma}$ is the stress tensor, and $\rho$ is the material density. Hooke's law relates the stress tensor $\sigma$ to strain tensor $\epsilon$ by

$$
\sigma_{i j}=K \epsilon_{k k} \delta_{i j}+2 \mu\left(\epsilon_{i j}-\frac{1}{3} \epsilon_{k k} \delta_{i j}\right), \quad i, j=1,2,3,
$$

where $K$ and $\mu$ are the bulk and shear moduli, respectively, and the use of subscript $k$ follows the Einstein summation convention. The strain-displacement relations are given by

$$
\epsilon_{i j}=\frac{1}{2}\left(\frac{\partial u_{i}}{\partial x_{j}}+\frac{\partial u_{j}}{\partial x_{i}}\right), \quad i, j=1,2,3 .
$$

\subsubsection{Boundary and Interface Conditions}

We have a boundary condition at the surface $\left(x_{3}=0\right)$ (for only BP5) and an interface condition on the fault $\left(x_{1}=0\right)$. At the free surface, all components of the traction vector are zeros, namely

$$
\sigma_{j 3}\left(x_{1}, x_{2}, 0, t\right)=0, \quad j=1,2,3 .
$$

Since the fault is always under compression in these benchmarks, there is no opening on the fault, namely:

$$
u_{1}\left(0^{+}, x_{2}, x_{3}, t\right)=u_{1}\left(0^{-}, x_{2}, x_{3}, t\right) .
$$

We define the slip vector as the jump in horizontal and vertical displacements across the fault:

$$
s_{j}\left(x_{2}, x_{3}, t\right)=u_{j}\left(0^{+}, x_{2}, x_{3}, t\right)-u_{j}\left(0^{-}, x_{2}, x_{3}, t\right), \quad j=2,3,
$$

with right-lateral motion yielding positive values of $s_{2}$. Positive values of $s_{3}$ and $s_{2}$ occur when the "+" or "-" side of fault moves in the positive or negative $x_{3}$ and $x_{2}$ directions, respectively.

We require that components of the traction vector be equal across the fault, which yields the following conditions:

$$
\sigma_{j 1}\left(0^{+}, x_{2}, x_{3}, t\right)=\sigma_{j 1}\left(0^{-}, x_{2}, x_{3}, t\right), \quad j=1,2,3,
$$

and denote the common values $-\sigma_{11}, \sigma_{21}$, and $\sigma_{31}$ by $\sigma_{\mathrm{n}}$ (positive in compression), $\tau_{y}$, and $\tau_{z}$, respectively, i.e. one normal traction component and two shear traction components. Note that positive values of $\tau_{y}$ indicate stress that drives right-lateral faulting and positive values of $\tau_{z}$ indicate stress that tends to cause the "+" side of the fault to move downward in the positive $x_{3}$ direction and the "-" side to move upward. 
We define the slip rate vector $\boldsymbol{V}$ in terms of its components, $\boldsymbol{V}=\left(V_{2}, V_{3}\right)=\left(\dot{s}_{2}, \dot{s}_{3}\right)$, where the dot notation indicates the time derivative, and denote slip rate amplitude as the norm of the slip rate vector, $V=\|V\|$. The shear stress vector is given by $\tau=\left(\tau_{y}, \tau_{z}\right)$.

In both benchmark problems, we assign a frictional domain on the fault, $\Omega_{f}$, with dimensions of $\left(L_{\mathrm{f}}, W_{\mathrm{f}}\right)$ in the along-strike and along-dip directions, where fault slip is governed by a rate- and state-dependent friction law (Dieterich, 1979; Ruina, 1983; Marone, 1998). The shear stress on the frictional fault $\tau$ is set to always equal the frictional strength $\boldsymbol{F}=\left(F_{2}, F_{3}\right)$, namely

$$
\boldsymbol{\tau}=\boldsymbol{F}\left(\bar{\sigma}_{\mathrm{n}}, \boldsymbol{V}, \theta\right),
$$

where the effective normal stress is $\bar{\sigma}_{\mathrm{n}}=\sigma_{\mathrm{n}}-p$, with normal stress $\sigma_{\mathrm{n}}$ and pore pressure $p$, and $\theta$ is a state variable.

For quasi-dynamic problems (BP4-QD and BP5-QD), $\boldsymbol{\tau}=\boldsymbol{\tau}^{0}+\Delta \tau-\eta \boldsymbol{V}$ is the sum of the prestress $\tau^{0}$, the shear stress change due to quasi-static deformation $\Delta \tau$, and the radiation damping approximation of inertia $\eta \boldsymbol{V}$ (Rice, 1993), where $\eta=\mu / 2 c_{\mathrm{s}}$ is half the shear-wave impedance for shear wave speed $c_{\mathrm{s}}=\sqrt{\mu / \rho}$, with the shear modulus $\mu$ and density $\rho$. For fully dynamic problems, $\tau=\tau^{0}+\Delta \tau$, where $\Delta \tau$ includes all elastodynamic stress transfers due to prior slip on the fault.

The frictional resistance of the fault is the product of the effective normal stress, $\bar{\sigma}_{\mathrm{n}}$, and evolving coefficient of friction, $f$, on the fault, namely

$$
\boldsymbol{F}\left(\bar{\sigma}_{\mathrm{n}}, \boldsymbol{V}, \theta\right)=\bar{\sigma}_{\mathrm{n}} f(V, \theta) \boldsymbol{V} / V
$$

The effective normal stress is taken to be uniform in space and unvarying in time, which is valid due to the symmetry across the planar fault and no fault opening. Since only the effective normal stress, not the normal stress, matters in Eq. 9, we use $\sigma_{\mathrm{n}}$ as a simpler notation for the effective normal stress in the remainder of this paper. We adopt a regularized formulation for the rate-and-state friction coefficient (Lapusta et al., 2000)

$$
f(V, \theta)=a \cdot \operatorname{arcsinh}\left[\frac{V}{2 V^{*}} \exp \left(\frac{f^{*}+b \ln \left(V_{*} \theta / D_{\mathrm{RS}}\right)}{a}\right)\right],
$$

where $D_{\mathrm{RS}}$ is the characteristic state evolution distance, $f^{*}$ is the reference friction coefficient determined at the reference slip rate $V^{*}$, and $a$ and $b$ are the parameters for the direct and evolution effects, respectively. We couple Eq. 10 with the aging law for the evolution of the state variable (Dieterich, 1979; Ruina, 1983):

$$
\frac{d \theta}{d t}=1-\frac{V \theta}{D_{\mathrm{RS}}},
$$

The spatial distributions of parameters $a$ and $b$ are chosen to create a seismogenic zone with velocity-weakening (VW; $a-b<0$ ) frictional properties that is surrounded by regions with velocity-strengthening (VS; $a-b>0$ ) frictional properties, with a linear transition zone in-between. We use the same 
value for parameter $b$ throughout the rate-and-state fault (denoted as $b_{0}$ ) and different values for parameter $a$ in the VW and VS regions (denoted as $a_{0}$ and $a_{\max }$, respectively).

Outside the frictional domain $\Omega_{f}$, we impose a fixed long-term fault slip rate, which we refer to as the plate loading rate $V_{\mathrm{L}}$, giving rise to the interface conditions:

$$
\begin{aligned}
& V_{2}\left(x_{2}, x_{3}, t\right)=V_{\mathrm{L}}, \\
& V_{3}\left(x_{2}, x_{3}, t\right)=0,
\end{aligned}
$$

At an infinite distance from the fault $\left(\left|x_{1}\right| \rightarrow \infty\right)$, the far-field displacements should follow:

$$
\begin{aligned}
& u_{2}^{ \pm}= \pm \frac{V_{\mathrm{L}} t}{2}, \\
& u_{1}=u_{3}=0,
\end{aligned}
$$

where the superscript " \pm " refers to the " +-- " sides of the fault, associated with positive and negative displacement values, respectively. By imposing this boundary condition, we consider displacements $\boldsymbol{u}$ that are only caused by slip, excluding the deformation that produced the prestress $\tau^{0}$ in the absence of fault slip. As a result, $\sigma$ are essentially stress changes associated with the displacement field $\boldsymbol{u}$ relative to the prestress state. For the fully dynamic problem, Eq. 13 must be augmented with radiation conditions that permit outgoing seismic waves (e.g., Bonnet, 1999). We describe an infinitely large domain in our benchmarks and leave choices of numerical implementation and approximation to modelers (see Section 3.1).

\subsubsection{Initial Conditions}

We choose the initial values of the stress and state on the fault to enable a spatially uniform distribution of initial fault slip rates, given by

$$
\boldsymbol{V}=\left(V_{\text {init }}, V_{\text {tiny }}\right) \text {, }
$$

where we assign $V_{\text {init }}=V_{\mathrm{L}}$ for simplicity and $V_{\text {tiny }}=10^{-20} \mathrm{~m} / \mathrm{s}$ to avoid infinity in logarithmic slip rates. To achieve this, we prescribe the initial state over the entire fault with the steady-state value at the slip rate $V_{\text {init }}$, namely

$$
\theta\left(x_{2}, x_{3}, 0\right)=D_{\mathrm{RS}} / V_{\text {init }}
$$

Accordingly, the initial stress vector takes the form $\tau^{0}=\tau^{0} \boldsymbol{V} / V$, where the scalar pre-stress $\tau^{0}$ is the steady-state stress:

$$
\tau^{0}=a \sigma_{\mathrm{n}} \cdot \operatorname{arcsinh}\left[\frac{V_{\text {init }}}{2 V^{*}} \exp \left(\frac{f^{*}+b \ln \left(V^{*} / V_{\text {init }}\right)}{a}\right)\right]+\eta V_{\text {init }} .
$$

For quasi-dynamic problems, we need to specify an initial value for slip, which we take to be zero, namely

$$
s_{j}\left(x_{2}, x_{3}, 0\right)=0, \quad j=2,3 .
$$


For fully dynamic problems, initial values for displacements and velocities in the medium need to be specified. We spare the details here since our code comparisons below will be limited to quasi-dynamic problems BP4-QD and BP5-QD.

To break the lateral symmetry of the fault and facilitate code comparisons, we add a square zone within the VW region, with a width of $w=12 \mathrm{~km}$ and a center at $(-22.5 \mathrm{~km},-7.5 \mathrm{~km})$ in BP4 and $(-24 \mathrm{~km},-10 \mathrm{~km})$ in BP5, as a prescribed nucleation location for the first simulated earthquake. To do that, we impose a higher initial slip rate, $V_{\mathrm{i}}$, in the $x_{2}$ direction within this square zone at $t=0$, while keeping the initial state variable $\theta\left(x_{2}, x_{3}, 0\right)$ unchanged. The resultant higher pre-stress is calculated by replacing $V_{\text {init }}$ with $V_{\mathrm{i}}$ in Eq. 16 . This initial condition leads to an immediate initiation of the first event. In BP5, we additionally use a smaller characteristic state evolution distance $D_{\mathrm{RS}}$ in this prescribed nucleation zone to promote the nucleation of subsequent earthquakes in the same areas (see the next section). We note that future benchmarks can use a spatially smoother function of the physical properties within the nucleation zone to minimize the influence of spatial discretizations in numerical models (Galis et al., 2015).

In simulations, the governing equations, Eqs. 1-3, are solved along with interface conditions, Eq. 4 (for only BP5) and Eqs. 5-13, and initial conditions, Eqs. 14-17, over the period $0 \leqslant t \leqslant t_{\mathrm{f}}$, where $t_{\mathrm{f}}$ is the maximum simulated time. Numerical methods that truncate model domain in the fault-normal direction also need to explicitly incorporate the far-field boundary conditions on asymptotic behavior of displacements at infinity (see Section 3.1). All model parameters in benchmarks BP4-QD and BP5-QD are listed and compared in Table 1.

\subsubsection{Critical Physical Length Scales}

Numerical resolution is a critical issue for 3D benchmark problems, as we need to balance the computational cost and adequate resolution to achieve acceptable model agreement. Two physical length scales are generally important to consider in these problems. The first length scale, often referred to as the process zone or cohesive zone, $\Lambda$, describes the spatial region near the rupture front under which breakdown of fault resistance occurs, and shrinks as ruptures propagate faster (Freund, 1990; Palmer and Rice, 1973). For faults governed by the rate-and-state friction, the quasi-static process zone at a rupture speed of $0^{+}, \Lambda_{0}$, can be estimated as follows (Day et al., 2005; Lapusta and Liu, 2009):

$$
\Lambda_{0}=C \frac{\mu D_{\mathrm{RS}}}{b \sigma_{\mathrm{n}}}
$$

where $C$ is a constant of order 1 .

The second length scale that controls model behavior is the nucleation size $h^{*}$, which determines the minimum size of the velocity-weakening region over which spontaneous nucleation may occur (Ampuero and Rubin, 2008; Rice and Ruina, 1983; Rubin and Ampuero, 2005). For 3D problems, 
the nucleation size can be estimated for the aging law for $0.5<a / b<1$ as follows (Chen and Lapusta, 2009):

$$
h^{*}=\frac{\pi}{2} \frac{\mu b D_{\mathrm{RS}}}{(b-a)^{2} \sigma_{\mathrm{n}}} .
$$

Using Eqs. 18 and 19, we estimate that the nucleation size is $12.4 \mathrm{~km}$ and $12.5 \mathrm{~km}$ within the VW region (outside the zone of frictional heterogeneity) in BP4 and BP5, respectively, whereas the process zone is 2 and $6 \mathrm{~km}$, respectively. This allows us to suggest $500 \mathrm{~m}$ and $1000 \mathrm{~m}$ for the grid spacing, $\Delta x$, in low-order accurate methods for BP4 and BP5, respectively, which resolve $\Lambda_{0}$ with at least four cells in both benchmarks, following suggestions by Day et al. (2005).

The two benchmark problems are designed to produce a periodic sequence of spontaneous earthquakes and slow slip, following the first event in which we impose higher local slip rates to kickstart the earthquake rupture. BP5 is slightly different from BP4 in that the characteristic state evolution distance $D_{\mathrm{RS}}$ is reduced within a square zone within the VW region, resulting in a smaller nucleation size, $h^{*}=11.6 \mathrm{~km}$. This form of persistent frictional heterogeneity is introduced to favor (but not always determine) the initiation of subsequent earthquakes at the same location. We choose the total simulated time to produce up to eight large earthquakes in the simulations, which allows us to examine not only a few early events but also the seismic behavior of the fault in the longer term.

\subsection{Model Outputs}

To assess model behavior over disparate spatial and temporal scales, we design several types of simulation outputs for these benchmarks (Figure 3): (1) time series of local on-fault and off-fault properties, (2) time series of global source properties, (3) a catalog of earthquake characteristics, (4) profiles of slip accumulation and stress evolution, and (5) rupture times during the first event in the sequence. The output formats for coseismic observables follow the practice in the code verification of single-event dynamic rupture simulations (Harris et al., 2009).

For local time series data, we are interested in resolving the time evolution of fault slip rates, shear stress, and off-fault displacements throughout the coseismic, postseismic, and interseismic periods. The global source properties refer to the evolving maximum slip rates and moment rates over the entire seismogenic fault areas, which are useful for determining the precise time of initiation and cessation of individual earthquakes. The catalog data contain key characteristics of simulated earthquakes, including their initiation and termination times, coseismic slip, and static stress drop. The beginning and end of the coseismic period are determined as the times at which any point on the fault reaches above or all points drop below a threshold slip rate, $V_{\text {th }}$ (chosen as $0.03 \mathrm{~m} / \mathrm{s}$ ), respectively. We then estimate coseismic slip and stress drop as the change in the amplitude of fault slip and shear stress (negative stress change corresponds to positive stress drop). 
The slip and stress profiles in the along-strike and along-dip directions illustrate the general patterns of earthquake sequences and the partitioning of seismic and aseismic slip. The rupture time data record the time when each point on the fault reaches a certain threshold slip rate $\left(V_{\text {th }}=\right.$ $0.03 \mathrm{~m} / \mathrm{s}$ ) during the first earthquake. Note that the relative rupture times are independent of $V_{\text {th }}$ and we can use maximum slip rates and rupture time data to construct contours of rupture fronts associated with different values of $V_{\text {th }}$.

\subsection{Modeling Groups}

To maximize participation, we focus on the quasi-dynamic version of the $3 \mathrm{D}$ benchmarks and anticipate new comparisons in future as the computational capabilities of the community grow. A total of 10 modeling groups participated in the code comparisons for the quasi-dynamic problems, BP4-QD and BP5-QD, using nine different numerical codes. We summarize numerical codes and methods, modeling groups, and their participation in either or both benchmarks in Table 2. Note that the simulations hosted on our online platform are named after the username of the modeler who uploaded the data; we include the names here for reference.

We discussed preliminary results of code comparisons for 3D benchmarks in two workshops in January and October 2020. We also used the opportunities to share scientific progress and decide on the directions of our future efforts, with substantial inputs from students and early career scientists. Our online platform (https://strike.scec.org/cvws/seas/) facilitates the initial comparison of benchmark results, where modelers can upload and immediately visualize time series data and rupture front contours to assess model agreements.

More modeling groups participated in BP5-QD than BP4-QD, due to considerations of timing and/or computational costs (Table 2). Given the similar problem setup of the two benchmarks, we present main results for BP4-QD and more complete comparisons for BP5-QD, using a selected suite of simulations listed in Tables 3 and 4. Several modelers have performed independent simulations using the same code (BICyclE and GARNET). These efforts ensure correct model setup and code execution and, in the case of BICyclE, expand the set of simulations and reveal the important effect of time stepping parameters (see Section 3.3). Due to limitations in code development and computational resources or different numerical methods, not all modeling groups have submitted all forms of requested simulation outputs. Our comparisons use the entire set of available simulation results.

\section{Computational Factors}

Both 3D SEAS benchmarks are computationally challenging: BP4-QD requires better numerical resolution and BP5-QD incorporates additional effects associated with the free surface. The overall high computational cost means that we have to carefully consider the effects of computational 
domain truncation and grid discretization on simulations that are performed near the marginal numerical resolutions. We elaborate on these computational factors in this section to provide important context to our code comparison results. We also comment on the time stepping schemes, an important ingredient in SEAS simulations.

\subsection{Domain Truncation and Boundary Conditions}

In the benchmark descriptions, we consider a whole space or semi-infinite half-space. All numerical codes need to truncate the computational domain in certain dimensions and adopt boundary conditions. While comprehensive tests about the effect of computational domain truncation and boundary conditions were conducted for our 2D benchmark problems (Erickson et al., 2020), they are less feasible for 3D SEAS simulations due to the much higher computational demand. We therefore let modelers determine sufficiently or reasonably large domain sizes using the suggested (or sometimes larger) grid spacing, with the aim of obtaining well-matching results. We denote the total model dimensions in the fault-normal, along-strike, and along-dip directions as $L_{1}, L_{2}$, and $L_{3}$, respectively (Figure 3a). The domain size of all simulations are listed in Tables 3 and 4 .

In general, BEM/SBEM simulations only discretize the fault interface and solve for on-fault physical properties, implicitly incorporating bulk response via analytical solutions. This feature avoids the need of domain truncation in the fault-normal direction; hence in Tables 3 and 4 we denote $\infty$ as the fault-normal dimension in BEM/SBEM simulations. Along lateral directions, BEM simulations with FDRA include three large elements outside the friction-controlled domain to construct semi-infinite loading zones of a dimension of $10^{4} \mathrm{~km}$. BEM simulations with ESAM, HBI, TriBIE, and Unicyle adopt same- or similar-sized elements and incorporate deep creep in the semi-infinite domain via a commonly used "backslip" approach, in which stress transfers are calculated for spatially-varying fault slip rates subtracted with $V_{\mathrm{L}}$. Hence the down-dip dimensions in these simulations are effectively infinite, even though we list the actual dimension of the adopted computational domain in Tables 3 and 4.

BEM/SBEM simulations with ESAM, BICyclE, and Motorcycle adopt periodic boundaries that effectively involve infinite replicas of the model domain in the along-strike direction; large areas with the imposed loading rate were included to minimize the effect of adjacent fault replicas on simulated fault behavior. Simulations with BICyclE also have periodic boundary conditions in the along-dip direction and, in the half-space problem BP5, approximate the free surface by adding a mirror image of the physical domain. Nonetheless, in our comparisons we do not observe systematic differences between BICyclE and other simulations, which suggests that the effect of these approximations is comparable to or smaller than other sources of discrepancies between different simulation methods. 
For volume-discretized methods such as EQsimu and GARNET, modelers need to truncate model domains in all three dimensions. For the far-field boundaries in the fault-normal direction, EQsimu and GARNET simulations use a Dirichlet boundary conditions for displacements via a fixed slip rate. When truncated fault-normal dimensions are not sufficiently large, the results are quantitatively influenced by this boundary condition. In BP5-QD, EQsimu modelers choose the steady interseismic velocity predicted by $V_{2}\left(x_{1}\right)=V_{\mathrm{L}} / \pi \cdot \arctan \left(x_{1} / D\right)$ (Savage and Burford, 1973), specifically, $V_{2} \approx 4 \times 10^{-10} \mathrm{~m} / \mathrm{s}$ (with $D=18 \mathrm{~km}$ and $x_{1}=L_{1} / 2=50 \mathrm{~km}$ ), to impose displacement boundary conditions in the far field. Both EQsimu and GARNET impose stress-free conditions at the remaining boundaries of the truncated domain, which includes two planes perpendicular to the fault and the bottom layer.

With computational resources as the limiting factor, these different approaches are in principle compatible with the boundary conditions at infinity as outlined in our benchmark descriptions. In our code comparison exercises, we will consider the effects of domain truncation and boundary conditions, especially for marginally resolved simulations.

\subsection{Grid Discretization}

The two benchmarks, especially BP5-QD, have a relatively large grid spacing by design, which is a nontrivial factor when we compare different simulations. For example, different codes represent local fault properties within piece-wise constant (BEM) or piece-wise linear (FEM) elements, or on Fourier sample points (SBEM). Most BEM codes use rectangular elements, whereas TriBIE uses triangular elements with their centroids on irregular grids. Additionally, FDM code GARNET uses a fully staggered grid, which means that velocities are not located on the same grid points with some other properties. Consequently, the computational grid points in these simulations are often offset from the observational points specified in the benchmark description. Even though these numerical codes are designed to solve the same continuum problem, different discrete representations of local physical properties, when combined with a relatively large grid spacing, result in nontrivial truncation errors that are different among these codes.

During early code comparisons for BP5-QD, we noticed that a spatial offset in the computational grid can lead to noticeable differences in the location and size of the prescribed nucleation region and rupture front development during the first event. Even though we have improved the consistency in model setups through several iterations among modelers, the inherent differences in computational methods continue to contribute to the discrepancies in the simulated outcomes. While this issue does not substantially affect our 2D benchmark problems (Erickson et al., 2020), it appears important in the comparisons for our 3D benchmarks, likely due to the use of larger cells. 


\subsection{Time Stepping Schemes}

The scheme of non-uniform, adaptive time stepping is essential in SEAS simulations that resolve various phases of earthquake source processes. We do not cover this computational aspect in the benchmark description and presume that modelers will adopt the optimal time stepping schemes for their numerical codes. Most codes use adaptive Runge-Kutta methods for time stepping. FDM code GARNET uses a linear multistep method (BDF2, second order backward differentiation formula) for their time stepping (Pranger, 2020). SBEM code BICyclE determines the adaptive time steps based on maximum slip rates and stability conditions derived from constitutive laws (Lapusta et al., 2000), which is also adopted in other codes, such as EQsimu and GARNET.

In practice, suboptimal time stepping can complicate model comparisons. In earlier comparisons for BP4-QD, one BICyclE simulation (jiang, denoted as BICyclE-1 hereinafter) exhibited frequent aseismic transients prior to large events, while these features were absent in another BICyclE simulation (lambert, denoted as BICyclE-2 hereinafter). We later tracked down the cause of this discrepancy: the latter simulation adopts a smaller constant factor in estimating the time step size (Eq. 18 in Lapusta et al. (2000)) and the use of finer time steps eliminates the aseismic transients, which are apparently numerical artifacts. We encountered a similar situation with EQsimu simulations, where a simple refinement of all time steps removed numerical transients and improved model agreement. Since we have corrected this issue in updated models, the choice on time stepping approaches should have a minimal influence on the comparison results presented below.

\section{Comparisons of 3D Simulations}

We examine a range of simulation outputs in the two benchmarks to understand model sensitivities and verify different numerical codes. We first show the agreement and self-convergence of models in BP4-QD (Figures 4-6), followed by more complete comparisons for BP5-QD (Figures 4 and 7-17). These comparisons include the rupture fronts of the first earthquake in the sequence (Figures 4 and 7), the long-term fault behavior in terms of maximum slip rates and earthquake characteristics (Figures 5 and 9), cumulative slip profiles (Figures 6 and 8), on-fault local stress and slip rate evolution in the long term (Figures 10 and 11) and during the coseismic period (Figures 12 and 14), as well as off-fault displacement behavior (Figure 15). Furthermore, we explore the relationship between interseismic stressing history and earthquake recurrence intervals (Figure 16) and the resolvability of coseismic observables in simulations with different spatial resolutions (Figure 17). 


\subsection{Whole-Space Problem BP4-QD}

\subsubsection{Initial Rupture Propagation}

The initial stage of the simulations provides a few crucial observables that are minimally affected by cumulating numerical errors. For benchmark BP4-QD, we first compare the coseismic rupture fronts during the first event in simulations with the suggested grid spacing ( $\Delta x=500 \mathrm{~m}$ ) (Figure 4a). We adopt a higher threshold slip rate than specified in the benchmarks, $V_{\mathrm{th}}=0.1 \mathrm{~m} / \mathrm{s}$, to define the initiation time of the earthquake as the moment when any point on the fault reaches $V_{\text {th }}$; we later explore how a different $V_{\text {th }}$ affects BP5-QD comparisons in Section 4.2.1. In Figure 4a, we find a discrepancy of $<1 \mathrm{~s}$ in local rupture arrival time (i.e., $<3 \%$ in average rupture speed) among simulations. We consider such a match of rupture fronts satisfying, given that the rupture arrival time has been shown to be a sensitive indicator of numerical precision in dynamic rupture simulations Day et al. (2005). The first simulated earthquake initiates within the prescribed nucleation zone and propagates outward through the rest of the VW region over a period of $\sim 30 \mathrm{~s}$. The suite of simulations with a grid spacing of $1000 \mathrm{~m}$ includes two volume-discretized codes. While the discrepancy in rupture times increases to a few seconds among all codes, the qualitative rupture pattern is unchanged in the coarser-resolution simulations.

\subsubsection{Long-term Fault Behavior}

We then assess the long-term fault behavior, in terms of maximum slip rates over the seismogenic fault areas, in simulations with different resolutions (Figure 5). The simulations with a $1000 \mathrm{~m}$ grid spacing come from a wider range of codes and show similar features of earthquake recurrence and interseismic periods, with fault slip rates varying between $\sim 10^{-9}$ and $1 \mathrm{~m} / \mathrm{s}$. Since the spatial model resolution is suboptimal, the simulations show a large variability in the transient aseismic slip between large earthquakes. These transient features are completely absent in simulations with a $500 \mathrm{~m}$ grid spacing and hence are numerical artifacts, rather than physical features. We also notice a persistent discrepancy of large event recurrence intervals which grows with the simulated time, even among better resolved simulations.

The computational demand of 3D benchmark problems prohibits a comprehensive self-convergence test of all participating numerical codes. We use the SBEM simulations (BICyclE-2) to demonstrate that self-convergence of simulation results may not show the true solution of the mathematically defined benchmark problems, when the domain size is not sufficiently large. In Figure 6, we show simulations with a range of grid spacings $(125,250,500$, and $1000 \mathrm{~m})$ and three computational domain sizes: $(120 \mathrm{~km}, 90 \mathrm{~km}),(240 \mathrm{~km}, 180 \mathrm{~km})$, and $(480 \mathrm{~km}, 360 \mathrm{~km})$ for the along-strike and along-dip model dimensions, denoted as S1, S2, and S3, respectively. 
The comparison of these simulations using the same code suggests challenges in assessing model agreement in 3D problems. First, with a smaller computational domain size (S1), simulations appear to converge to a similar pattern of long-term behavior (Figure 6a-b). However, when the computational domain size is increased ( $\mathrm{S} 2$ and S3), the simulations produce different earthquake patterns, with alternating nucleation locations (Figure $6 \mathrm{c}-\mathrm{d}$ ). This difference results in a minor, though noticeable, change in the recurrence time of subsequent events (Figure 6a). The sensitivity of nucleation location in BP4-QD likely stems from the spatially uniform frictional properties and near-symmetric stress field associated with the fault-spanning quasi-dynamic earthquake ruptures. Even though we are approaching the computational limit, we expect that model behavior will presumably stabilize and converge to the same pattern as domain size substantially increases, as we have seen in 2D problems (Erickson et al., 2020).

We note that, physically, these results arise since the two ends of the fault represent similar favorable nucleation locations in the uniform fault model setup, which allows minor computational changes to affect which nucleation location wins. This further implies that, on such a fault, minor outside perturbations (not modeled here), such as stress changes from slip on nearby faults, would determine the nucleation location. Note also that the incorporation of full wave-mediated inertial effects, not considered in this benchmark, are expected to create much larger differences in the model response based on prior studies (Lapusta and Liu, 2009; Thomas et al., 2014).

\subsection{Half-Space Problem BP5-QD}

\subsubsection{Initial Rupture Propagation}

The rupture fronts of the first event in BP5-QD simulations $\left(V_{\mathrm{th}}=0.1 \mathrm{~m} / \mathrm{s}\right)$ show a close match and slightly larger discrepancy compared with BP4-QD results, partly due to different grid spacings (Figure 4). The simulated earthquake rupture propagates into the transition zones around the VW region and reaches the surface, with the total rupture lasting over $30 \mathrm{~s}$. The maximum discrepancy in local rupture time is less than two seconds among most simulations (5-10\% discrepancy in rupture speeds), and a few seconds between the EQsimu simulation and others ( $10 \%$ discrepancy in rupture speeds) with the former showing higher rupture speeds.

When we use a lower threshold slip rate, $V_{\mathrm{th}}=0.03 \mathrm{~m} / \mathrm{s}$, to determine the coseismic phase, the rupture front contours appear more discrepant, though retaining a qualitative agreement (Figure 7a). This alternative comparison reveals a large variability in the evolution of slower slip preceding the earthquake rupture among simulations. We observe increased discrepancies among SBEM/BEM simulations, while the largest discrepancies are associated with the two volume-discretized codes, which seem to produce rupture speeds that are either higher or lower than the average values among 
the group. Nonetheless, a smaller grid spacing helps reduce the differences in rupture fronts between EQsimu and other simulations, albeit at an increased computational expense (Figure 7b).

\subsubsection{Long-Term Fault Behavior}

We first show the overall earthquake patterns in BP5-QD (Figure 8). We juxtapose the profiles of fault slip evolution in the along-strike and along-dip directions from two codes, FDRA and BICyclE, based on BEM and SBEM methods, respectively. The results show that, after the first earthquake, later events exhibit recurrent slip patterns. The coseismic slip initiates and propagates through the VW region and into the shallow VS region, whereas postseismic and interseismic slip occurs in the adjacent VS regions and to a lesser extent near the surface. In contrast to BP4-QD, BP5-QD simulations generally have a persistent location for earthquake initiation due to the heterogeneity in frictional properties that we introduce in this benchmark.

We find an overall good agreement of maximum slip rates over the seismogenic fault areas among simulations with the suggested resolution $(\Delta x=1000 \mathrm{~m})$ (Figure 9a). The inter-event times of simulated earthquakes vary around $\sim 235$ years over the 1800 -year simulation period. A small yet persistent difference in recurrence intervals leads to apparent divergent timing of large events in simulations, especially for the EQsimu simulation which exhibits some pre-event aseismic transients. Despite the minor discrepancy in rupture fronts shown earlier, the total rupture duration and static stress drop of the first event match closely among simulations where catalog data are available (Figure $9 \mathrm{~b}-\mathrm{c}$ ). We determine the beginning and end of the coseismic period as the times at which any point on the fault reaches above or all points drop below a threshold slip rate of $0.1 \mathrm{~m} / \mathrm{s}$, respectively, to be consistent with how we estimate the rupture time in Figure 4. The simulated earthquakes have robust characteristics, with rupture durations of $\sim 30 \mathrm{~s}$ and stress drops of $\sim 5 \mathrm{MPa}$.

We then examine the time evolution of local slip rates and shear stress on the fault, at the surface $\left(x_{3}=0 \mathrm{~km}\right)$ and the mid-seismogenic depth $\left(x_{3}=10 \mathrm{~km}\right)$, during the first 1000 years of BP5-QD simulations (Figures 10 and 11). The periodic variations in local shear stress and slip rates are distinct at different depths. At the surface, the fault creeps with slip rates comparable to the plate rate before dynamic rupture comes (Figure 10b, d), and hence the rapid increase of slip rates to $\sim 1 \mathrm{~m} / \mathrm{s}$ at the rupture front results in a large direct effect on the shear stress (the vertical lines in Figure 10a, c), amplified by the large value of the rate-and-state parameter there ( $a=0.04)$. At the same time, the smaller slip at the free surface due to its VS nature results in smaller static stress drops (the difference in shear stress before and after the vertical lines that represent dynamic rupture) of $\sim 1 \mathrm{MPa}$.

In contrast, substantial static stress drops of $\sim 10 \mathrm{MPa}$ occur within the VW region during earthquakes, followed by interseismic strain buildup, leading to slip rate variations over tens of 
orders of magnitude (Figure 11). The direct effect during the dynamic rupture appears weaker at mid-seismogenic depth than the surface, due to the smaller value of rate-and-state parameter $(a=0.004)$. We observe a slightly larger discrepancy between simulations at depth than at the surface. Despite noticeable differences in earthquake recurrence times, all simulations accurately capture the full range of slip rate and stress variations. While simulations performed at the suggested resolution $(\Delta x=1000 \mathrm{~m})$ already show good agreements in terms of the long-term fault behavior, a smaller grid spacing $(\Delta x=500 \mathrm{~m})$ further improves the results.

\subsubsection{Coseismic Rupture and Off-Fault Behavior}

The comparisons of individual earthquake ruptures show consistency of different simulations, as well as complexity in the location and development of earthquake nucleation. In Figure 12, we show time evolution of slip rates and shear stresses during the first simulated event at three representative locations on the fault: within the prescribed nucleation zone $\left(x_{2}=-24 \mathrm{~km}, x_{3}=10 \mathrm{~km}\right)$, at the surface $\left(x_{2}=x_{3}=0 \mathrm{~km}\right)$, and within the rupture propagation zone $\left(x_{2}=0 \mathrm{~km}, x_{3}=10 \mathrm{~km}\right)$. All time series data are aligned relative to the earthquake initiation time (defined with a threshold slip rate $V_{\text {th }}=0.1 \mathrm{~m} / \mathrm{s}$ ) in each simulation. Consistent with Figures $4 \mathrm{~b}$ and 7 , all simulations show excellent agreement of the temporal functions of slip rates and shear stresses, with minor differences in rupture arrival times and peak slip rates.

For the simulated fourth event, we find slightly increased model discrepancies, due to subtle differences in the earthquake nucleation condition resulting from the prior slip history (Figure 13). While most simulations retain the same source evolution function, the results from two simulations with TriBIE and EQsimu appear qualitatively different over much of the seismogenic zone. This pronounced difference is due to the different initiation locations of the earthquake, similar to the results in Figure 6. With a large nucleation zone in BP5-QD, much of the deeper VW zone hosts aseismic slip in the interseismic period. These areas can serve as alternative locations to start an earthquake, when the local stress conditions near the transition from VS to VW fault regions outcompete the processes in the prescribed nucleation zone. When we compare simulations with a halved grid spacing of $500 \mathrm{~m}$, the variability of nucleation location in TriBIE and EQsimu simulations disappears. The distinct behavior of these simulations based on BEM and FEM methods suggests that the earthquake nucleation in this benchmark is still susceptible to the specific setup of a computational model.

To further assess model convergence, we compare the sixth event in simulations with smaller grid spacings, including most simulations at $500 \mathrm{~m}$, and BICyclE-2 and ESAM simulations at both 500 and $250 \mathrm{~m}$ resolutions (Figure 14). Simulations with a grid spacing of $500 \mathrm{~m}$ show nearly identical source time function with small time offsets, an overall excellent agreement. However, some codes again display nucleation at the other end of the fault. Similar to the aforementioned results about TriBIE and EQsimu, we find that earthquake nucleation in finer-resolution simulations 
(250 m) with BICyclE return to the same location that matches other simulations. In spite of such variability in a few simulations, the clear improvements in model agreement suggest that different numerical codes will likely converge to the same behavior with a decreased grid spacing.

We also compare the off-fault behavior in simulation groups where these outputs are available (Figure 15). Note that most of these simulations explicitly solve for off-fault responses, whereas the off-fault displacements for BICyclE-2 are computed from previously simulated fault slip history and analytical Green's functions (Okada, 1992). For Unicycle and TriBIE, off-fault displacements are calculated in the simulations using Okada Green's functions for only fault patches in the frictional domain, excluding deep-seated displacements. For a consistent comparison with other simulations, we add long-term displacement trend to off-fault time series from Unicycle and TriBIE simulations using $V_{2}\left(x_{1}\right)=V_{\mathrm{L}} / \pi \cdot \arctan \left(x_{1} / D\right)$ (Savage and Burford, 1973), where we assume a locking depth $D$ of $18 \mathrm{~km}$.

Focusing on the first and fourth event, we observe a good qualitative agreement of surface velocity time series at various distances away from the fault, with the fourth event more challenging to match (Figure 15a-b). Overall, the discrepancies in coseismic off-fault deformation appear larger than all the on-fault properties that we have examined. This is likely due to multiple factors, including inaccurate representations of surface observation points (e.g., grid points offset from the surface) and domain truncation in the fault-normal direction. The long-term displacement histories at these off-fault locations also yield good qualitative agreements (Figure 15c).

\subsubsection{Model Discrepancy and Convergence}

From previous comparisons, we observe that long-term model observables such as recurrence intervals appear more variable than short-term earthquake characteristics such as coseismic slip and stress drop. To better understand the long-term divergence of simulation results, we examine the interseismic stressing history and its relationship with earthquake recurrence intervals (Figure 16). We first calculate the changes in shear stress within the seismogenic zone in the postseismic and interseismic period leading up to the sixth event. The mid-seismogenic stressing history features higher positive stressing in the early postseismic period due to decaying afterslip, followed by increasing positive stressing in the later interseismic period and negative stressing as the creep fronts enter the seismogenic zone. We can estimate the minimum stressing rate (in insets of Figure 16a, c) when the postseismic period transitions to the interseismic period. This minimum stressing rate is well-defined and less susceptible to the complex fault slip history, hence reflecting differences in large-scale, long-term loading in each simulation.

In both simulation groups using grid spacings of 1000 and $500 \mathrm{~m}$, we find that the minimum interseismic stressing rate is approximately inversely correlated with the nearly constant recurrence 
intervals of large events (Figure 16b, d). This minimum stressing rates in volume-discretized codes EQsimu and GARNET tend to deviate from the cluster of SBEM/BEM results, although the general relationship between interseismic stressing rates and recurrence intervals still holds. The subsequent stressing history appears more variable among many simulations, especially in cases with a grid spacing of $500 \mathrm{~m}$, indicating the complexity in aseismic slip evolution. These comparisons suggest that stress buildup process is essentially similar across simulations and explain why these simulations have more robust earthquake characteristics, even in the presence of growing discrepancies in the long term.

We then characterize the convergence of these simulations with different numerical resolutions, in terms of three observables of simulated earthquakes (Figure 17). We plot the total rupture duration, and final slip and peak slip rate at the center of the VW region $\left(x_{2}=0 \mathrm{~km} ; x_{3}=10 \mathrm{~km}\right)$ during the first and sixth events, because these quantities capture the overall or local properties of earthquake ruptures. We have included BEM/SBEM simulations with resolutions from $2000 \mathrm{~m}$ down to $250 \mathrm{~m}$, and FEM/FDM simulations with a smallest grid spacing of 500 or $1000 \mathrm{~m}$. We see a better agreement in these observables for the first event than the sixth event and a closer match in simulations with smaller grid spacings, consistent with our earlier results (Figures 4, 12, and 14). As the convergence test of simulations are not always computationally feasible for these 3D problems, these comparisons provide an alternative approach to verify the involved numerical codes.

\section{Discussion}

\subsection{Important Computational and Physical Factors}

The dominant factor controlling the response of the model is the numerical resolution (grid spacing or cell size). While this is not surprising, our results show that marginal resolution significantly affects the results. SEAS simulations are often done on the boundary of resolution, especially in $3 \mathrm{D}$, due to substantial computational costs and the desire to consider realistic physical properties. Our BP4-QD simulations show that the marginal cell size of $1000 \mathrm{~m}$ (which still resolves the quasi-static process zone by 2 cells and the nucleation zone by $\sim 12$ cells) captures the main qualitative aspects of the fault response but results in significant quantitative differences with the better-resolved simulations, including much different recurrence time, larger discrepancies between different simulation approaches, and artificial slow-slip transients for some codes. Reducing the cell size even to $500 \mathrm{~m}$ results in significant improvement, with a closer match between different simulation codes.

For the adequate numerical resolution, we find that further differences occur due to the choice of the computation domain and the associated discrepancy in the boundary and loading conditions simulated. The comparisons of global fault properties in BP4-QD (Figures 5 and 6) demonstrate that simulations with the same code (BICyclE) produce robust earthquake patterns and properties 
with the decrease in grid spacing. However, the apparent self-converging behaviors are associated with specific domain sizes. The model discrepancy persists due to the variability of earthquake nucleation locations, even when we adequately resolve the cohesive zone during rupture propagation with a grid spacing of 125, 250 and $500 \mathrm{~m}$. These results for BP4-QD suggest that domain truncation prevents simulations from converging toward the solution to the semi-infinite domain problem, at least with current computational resources.

Practically, this effect is relatively small in our study compared with the differences between simulations with adequate and inadequate numerical resolutions. Since there is a trade-off between large computational domains and fine numerical resolution, caution should be exercised when modelers expand the domain size at the expense of numerical resolution. While we have explored the domain effect in SBEM simulations which are relatively efficient and allow us to choose larger domain sizes, similar considerations would apply to simulations using BEM/FEM/FDM methods.

Rupture front contours are diagnostic of rupture behavior and hence a key metric for model agreement, as noted for single-event dynamic rupture simulations (Barall and Harris, 2015; Harris et al., 2009). In SEAS simulations, many factors can lead to large discrepancies in rupture fronts even for the first event. Some issues are fixable, such as inaccurate or inconsistent model setup and parameter choices (Section 3). Some factors can be mitigated in improved benchmark design. For example, when revising BP5-QD, we increased the elevated initial slip rate, $V_{\mathrm{i}}$, in the prescribed nucleation zone from $0.01 \mathrm{~m} / \mathrm{s}$ to $0.03 \mathrm{~m} / \mathrm{s}$. This change shortens the period of pre-rupture stress buildup which turns out to be sensitive to the domain size, and improves agreement of the first simulated earthquake rupture..

We notice inherent challenges in achieving agreements among simulations when the free surface is present. The comparison between BP4-QD and BP5-QD simulations with a grid spacing of $500 \mathrm{~m}$ (Figures $4 \mathrm{a}$ and $7 \mathrm{~b}$ ) suggests that the presence of the free surface and its interaction with earthquake rupture contribute to increased model discrepancies, even though the cohesive zone is better resolved (by more cells) in BP5-QD. Since we do not have simulations for the exact BP5-QD model setup in both whole space and half-space, we cannot directly characterize the effect of the free surface on 3D benchmark results.

Understanding the impact of heterogeneities in SEAS models is important for both benchmark design and code comparison. The prestress of earthquake ruptures depends on prior fault slip history and varies in space, even in the case of uniform frictional properties in BP4-QD. When designing the BP5-QD problem, we introduced persistent frictional heterogeneity to promote earthquake initiation at the same location, thereby largely avoiding the difficulty in comparing individual events in BP4-QD. However, in some simulations, prestress heterogeneity can still outcompete the frictional heterogeneity to result in different earthquake rupture patterns (Figures 13 and 14). These complexities 
in the physical problem help reveal the subtle differences of numerical codes but also impose challenges on our efforts to define and pursue successful code verification.

\subsection{From 2D to 3D Benchmarks}

The experience and findings from our code verification exercises for 2D SEAS benchmarks (Erickson et al., 2020) are indispensable for code comparisons of 3D SEAS models. Strict self-convergence tests are often feasible in 2D problems, allowing us to comprehensively explore how suboptimal choices of computational domain size and model resolution can affect earthquake recurrence intervals and event statistics. The findings from $2 \mathrm{D}$ benchmarks hence serve as essential reference examples when we grapple with the effects of various computational factors in challenging 3D problems.

Benchmark problems in 3D have several unique features. First, the computational constraint of $3 \mathrm{D}$ problems motivate us to design verification methods and metrics to reveal the relative sensitivities of different model observables near the marginal numerical resolutions. Specifically, earthquake rupture characteristics such as rupture duration, final slip, and peak slip rate appear to be more robust than other longer-term observables such as recurrence intervals and nucleation phase, because domain-size-dependent loading can substantially affect aseismic slip evolution. As expected, global fault properties are more robust than local fault behavior. Second, the 3D nature of the problem brings new physical complexity, in particular the multiple potential locations for earthquake nucleation, compared with the single downdip nucleation location in 2D antiplane problem (Erickson et al., 2020). The interactions of stress heterogeneity and frictional properties throughout the fault slip history ultimately control earthquake nucleation, which cannot be assigned a priori by modelers. Third, the 3D setting and the presence of a free surface enables a direct comparison of model results and more complicated geophysical observations, which is important for the efforts to validate SEAS models.

We highlight a few important outcomes of our code comparison results in connections to our 2D exercises. First, excellent quantitative agreements in key model observables can be achieved with proper numerical resolution among different modeling group. Second, at marginal resolutions, several factors combine to affect model agreements and convergence. For this reason, we find generally larger discrepancies among the earthquake ruptures in 3D SEAS simulations than those in 2D SEAS and 3D single-event dynamic rupture simulations. Third, even in well-resolved models, long-term model observables are more sensitive than earthquake observables to minor differences in computational factors.

\subsection{Implications for Model Validation}

Our successful code comparison exercises lend confidence to the accuracy of the participating numerical codes, serving as an essential step towards the goal of creating valid, physics-based 
models for earthquake source processes. In our benchmarks, many simulated physical quantities can be measured or inferred using geological and geophysical observations covering disparate spatial and/or temporal scales, such as seismograms, Global Navigation Satellite System (GNSS), satellite imagery, and paleoseismic records, offering opportunities for model validation. Furthermore, our efforts to understand how sensitive and variable model observables are to both computational and physical factors also contribute to quantifying and reducing uncertainty in the data-model integration. Ultimately, SEAS models validated with real-world observations could contribute to estimating earthquake hazard.

Despite computational challenges, the SEAS modeling framework presented here rigorously resolves the important spatial and temporal scales in earthquake source processes, in ways that are complimentary to and synergistic with dynamic rupture simulations and earthquake simulators. The computational rigor and realistic physical processes in SEAS modeling can help inform and improve the choices of procedures and parameterization, and approximation of physics in other modeling frameworks. Examples include the design of self-consistent pre-rupture stress conditions, and assessing the role of transient slow slip in time-dependent seismic hazard.

\section{Conclusions}

We present code comparison results for 3D models of earthquake sequences and aseismic slip from two recent benchmarks in the SEAS initiative (Erickson et al., 2020). The increased complexity and computational cost of 3D SEAS problems motivate us to adopt new strategies for benchmark design and code verification using a range of simulation outputs. We assess the contours of coseismic rupture fronts, time series of fault slip, slip rates, and shear stress, time series of off-fault displacement and velocity, and history of maximum fault slip rates, as well as earthquake catalogs, from tens of simulations contributed by 10 modeling groups.

We achieve excellent model agreements among most outputs and observables with relatively large computational domain size, although discrepancies are larger than those in 3D single-event dynamic rupture and 2D SEAS simulations, partly due to spatial resolutions limited by the computational cost. The successful code verification exercises lend confidence to the accuracy of participating numerical codes. The quantitative differences of simulation results depend on computational factors such as grid discretization and spacing, model domain size, and boundary conditions. Coseismic observables appear more robust than longer-term, aseismic observables that are more easily influenced by long-term accumulating numerical errors and domain-size-dependent loading. An important factor that can influence the interseismic behavior is the variable time stepping procedures, and exploring their effect on the larger discrepancy of the aseismic observables is an important direction of future work. Understanding the causes of model discrepancies and relative sensitivities of various 
model observables are important, as researchers work towards integrating numerical simulations with the increasing volumes of geological and geophysical observations.

The earthquake problem is a prime example of a dynamic solid Earth system that spans a wide range of spatial and temporal scales. Our community-driven code verification efforts are aimed at improving and promoting a new generation of rigorous, robust numerical codes for earthquake science. Our results and lessons could be useful to other research areas that involve numerical simulations of nonlinear, multi-scale dynamic problems.

\section{Open Research}

Descriptions of benchmarks BP4 and BP5 are available at https://strike.scec.org/ cVws/seas/download/ (SEAS_BP4_QD.pdf and SEAS_BP5.pdf) and included as supplementary materials. Our online platform (https://strike.scec.org/cvws/seas/) hosts the simulation data for local and global fault properties and rupture times. See publications in Table 2 for the availability and repositories of numerical codes. GARNET is available at https://bitbucket. org/cpranger/garnet/.

\section{Acknowledgments}

We thank Michael Barall for maintaining the SEAS online platform and Ruth Harris for providing experience from the SCEC/USGS code verification exercises for dynamic rupture simulations. We thank Steve Day, Ruth Harris, Associate Editor, and Editor for reviewing this manuscript. J.J. and B.A.E. designed the benchmark problems and organized the workshops for code verification exercises. J.J. analyzed all simulation results and led the writing of the manuscript. All remaining authors provided feedback on benchmark design, participated in the benchmark exercises (listed in Table 2), and/or helped with revising the manuscript. V.L. additionally helped with the early tests of benchmark problems; the other authors are listed alphabetically.

The work of J.J. and B.A.E. on this project and SEAS-themed workshops have been supported by the Southern California Earthquake Center (SCEC). This research is SCEC Contribution No. 11680. SCEC is funded by National Science Foundation (NSF) Cooperative Agreement EAR-1600087 and United States Geological Survey (USGS) Cooperative Agreement G17AC00047. The simulations with BICyclE (J.J.) were conducted with the award EAR170014 from the Extreme Science and Engineering Discovery Environment (XSEDE), which is supported by NSF Grant No. ACI-1548562. The simulations with BICyclE (V.L. and N.L.) were carried out on the High Performance Computing Center cluster of the California Institute of Technology with the grant support from SCEC. The simulations with Unicycle and Motorcycle are supported in part by the NSF Grant No. EAR-1848192. The simulations with ESAM are supported by Natural Sciences and Engineering Research Council of Canada (NSERC) Discovery Grant RGPIN-2018-05389. The simulations with HBI are supported 
in part by Japan Society for the Promotion of Science (JSPS) KAKENHI Grant No. 19K04031.The simulations with GARNET are supported by the Dutch Research Council (NWO) grant DEEP.NL.2018.037, Swiss National Science Foundation (SNSF) grant 200021-169880, EU-MC ITN grant 604713, and EU ERC StG 852992. The simulations with TriBIE are supported by the European Union's Horizon 2020 research and innovation program (TEAR ERC Starting Grant No. 852992) and NSF Grant No. EAR-2121666. The simulations with EQsimu were conducted with the advanced computing resources provided by Texas A\&M High Performance Research Computing and by the Texas Advanced Computing Center (TACC) at the University of Texas at Austin. 
A Model ingredients: e.g., surface and subsurface structure, rock properties, friction, and rheology

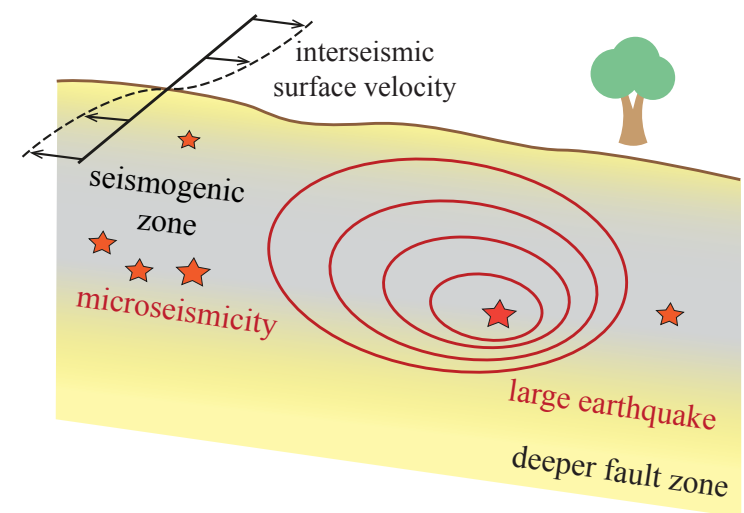

B Model outputs: e.g., dynamic rupture, ground shaking and displacements, and stress change

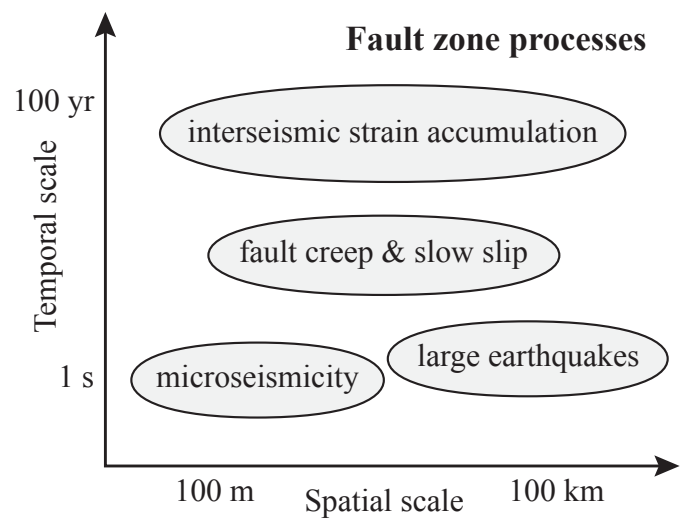

Figure 1. Main ingredients and outputs in 3D models of sequences of earthquakes and aseismic slip (SEAS).

(a) SEAS models incorporate the surface and subsurface structure, rock properties, friction, and rheology of a fault zone to simulate earthquakes and aseismic deformation. In the sketch of a strike-slip fault model, earthquake hypocenters are marked by red stars and rupture fronts of large earthquakes are shown as red contours. The seismogenic zone is colored in gray and aseismic fault zone in yellow. (b) SEAS models produce many outputs, including fault slip, off-fault displacements, and stress changes, which can be connected to observations of fault zone processes spanning a range of spatial and temporal scales, such as microseismicity, large earthquakes, fault creep, slow slip, and interseismic strain accumulation. 


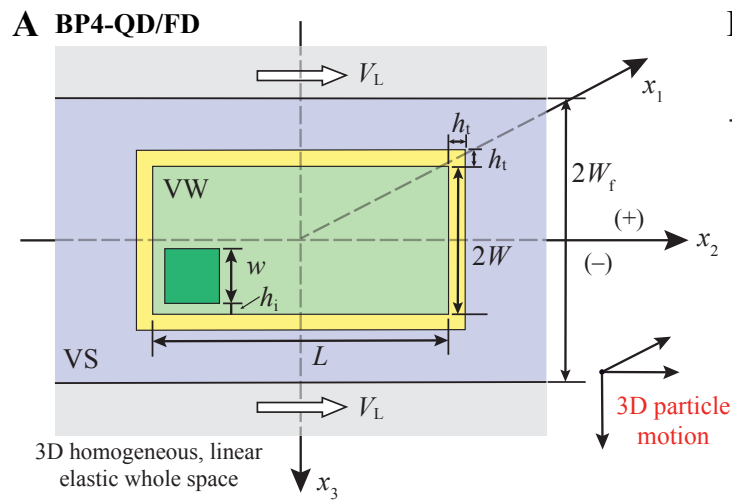

\section{B BP5-QD/FD}

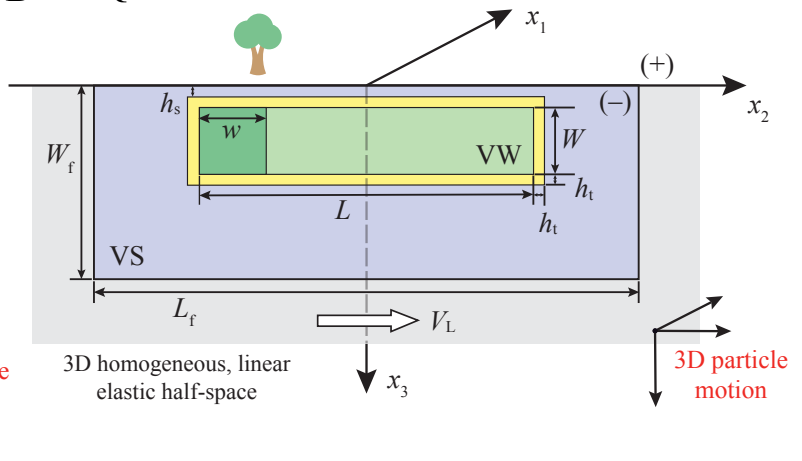

Figure 2. Two benchmark problems for 3D SEAS models. The benchmarks (a) BP4 and (b) BP5 consider 3D motion with a vertical planar fault embedded in a homogeneous, isotropic, linear elastic whole space and a half-space with a free surface, respectively. The fault is governed by a rate-and-state friction (RSF) law in the central region (non-gray colors) and assigned a constant rate at the boundaries (gray). The velocity-weakening (VW) region (light and dark green) is surrounded by a transition zone (yellow) and velocity-strengthening (VS) regions (blue). In the $x_{2}$ and $x_{3}$ directions, the sizes of the frictional domain and VW region are $\left(\infty, 2 W_{\mathrm{f}}\right)$ and $(L, 2 W)$, respectively, for BP4, and $\left(L_{\mathrm{f}}, W_{\mathrm{f}}\right)$ and $(L, W)$ for BP5. The initial nucleation zone (dark green square) is located at one end of the velocity-weakening region. Earthquakes spontaneously nucleate and propagate across the seismogenic fault. FD and QD in the benchmark names refer to fully dynamic and quasi-dynamic earthquake rupture problems, respectively. 
A Computational Model

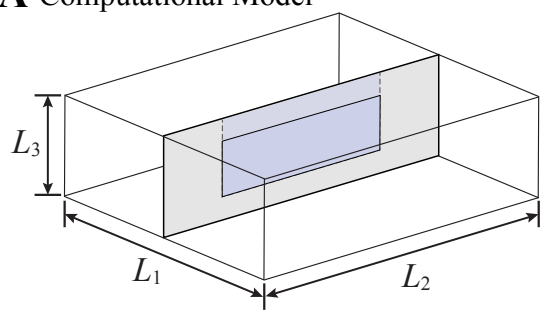

B On-fault stations in BP4-QD

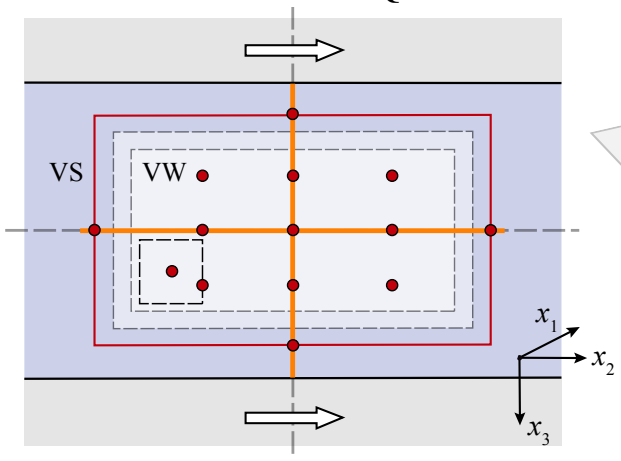

C On-fault stations in BP5-QD

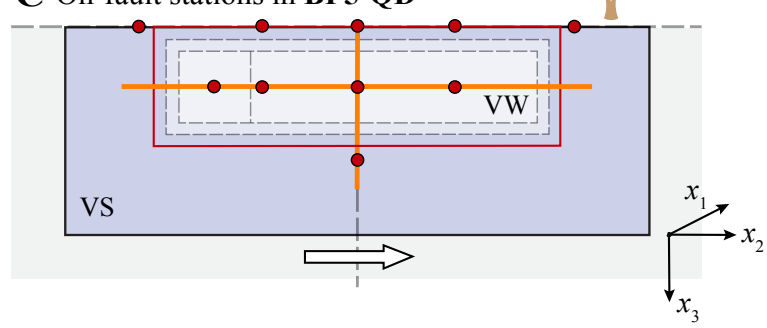

D Off-fault stations in BP5-QD

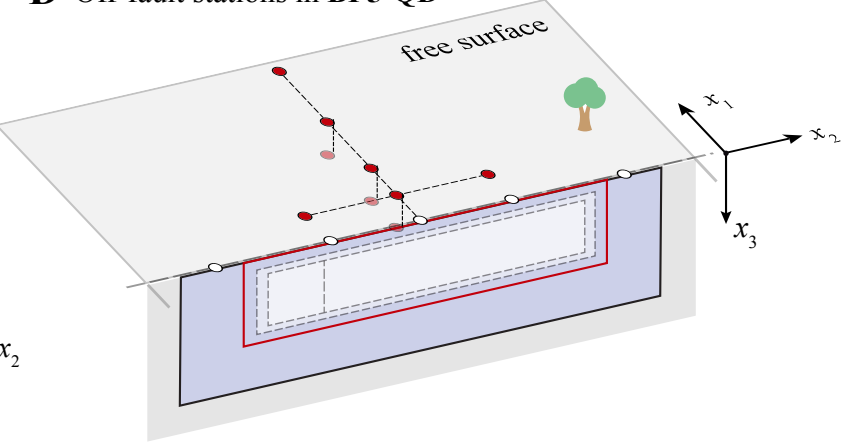

Figure 3. Computational model setup and simulation outputs for 3D SEAS benchmarks. (a) The fault-normal, along-strike, and along-dip dimensions of a computational model is denoted as $L_{1}, L_{2}$, and $L_{3}$, respectively. Observation points, lines, and areas are shown for (b) BP4 and (c and d) BP5. Local time series are produced at (b and c) on-fault and (d) off-fault points (red). Profiles of slip and stress evolution are produced along cross-section lines (orange). The region outlined in red is used to compute time-dependent source properties and rupture front contours. Dashed rectangles indicate fault areas with different frictional properties. 


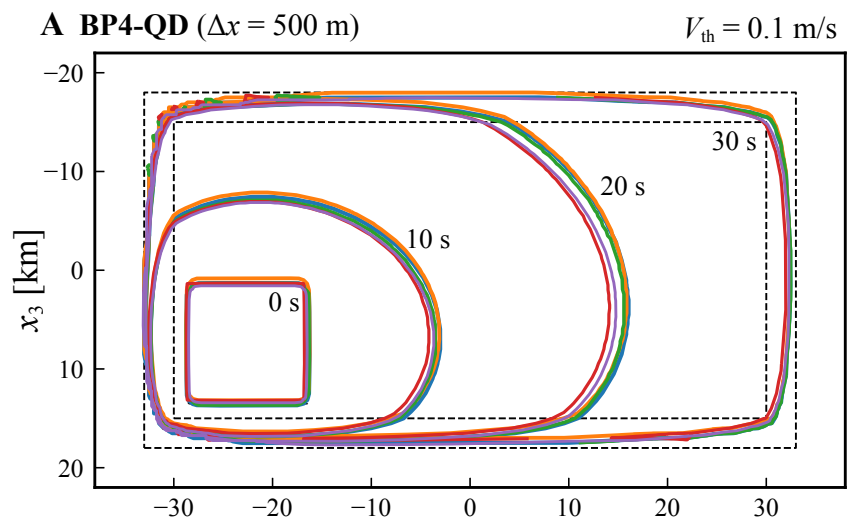

\section{BP4-QD}

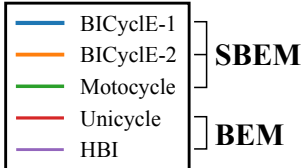

BP5-QD

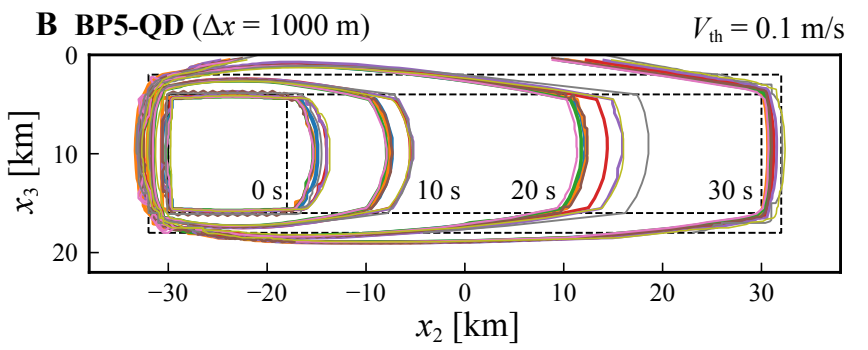

Figure 4. Rupture fronts of the first earthquake in BP4-QD and BP5-QD simulations with suggested numerical resolutions. The contours of rupture fronts are shown for simulations in (a) BP4-QD $(\Delta x=500 \mathrm{~m})$ and (b) BP5-QD $(\Delta x=1000 \mathrm{~m})$. The rupture front contours indicate $0,10,20$, and $30 \mathrm{~s}$ after the earthquake initiation time, defined as the moment any point on the fault reaches a threshold slip rate $V_{\mathrm{th}}=0.1 \mathrm{~m} / \mathrm{s}$. The legends show code names and corresponding types of numerical methods listed in Table 2. BICyclE-1 and BICyclE-2 refer to simulations from jiang and lambert, respectively. 

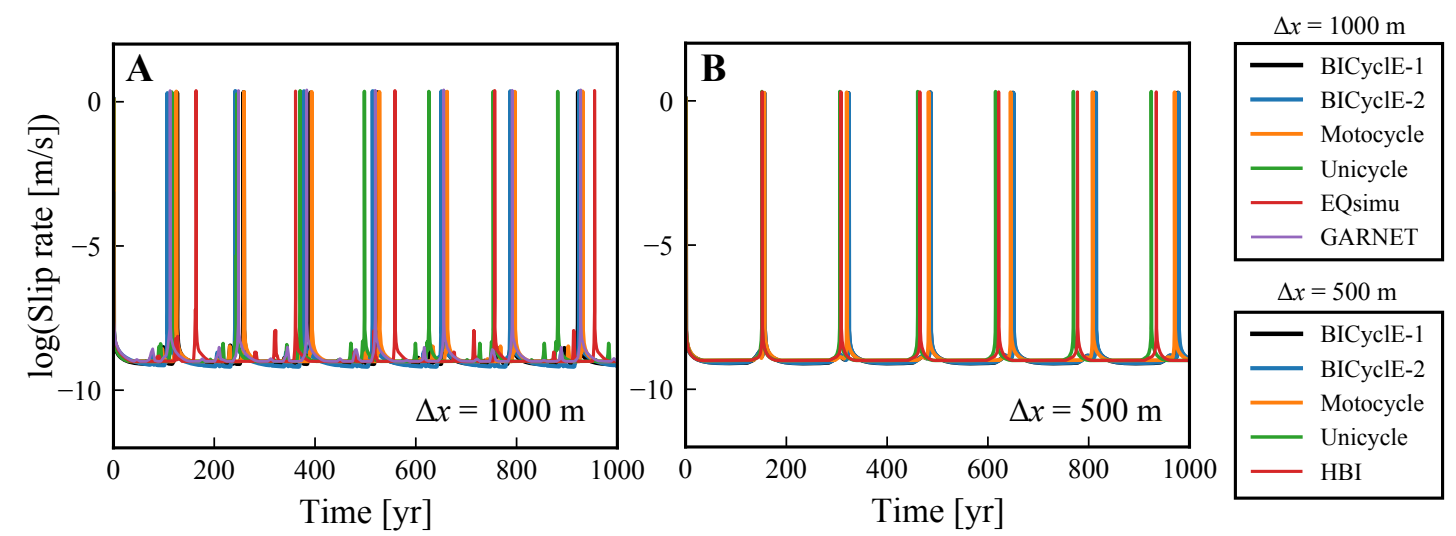

Figure 5. Time evolution of maximum slip rates in BP4-QD simulations. The time series of logarithmic maximum slip rates within the seismogenic zone are shown for simulations with (a) $\Delta x=1000 \mathrm{~m}$ and (b) $\Delta x=500$ $\mathrm{m}$. We use logarithms with base 10 and code names in legends in this and all later figures. 

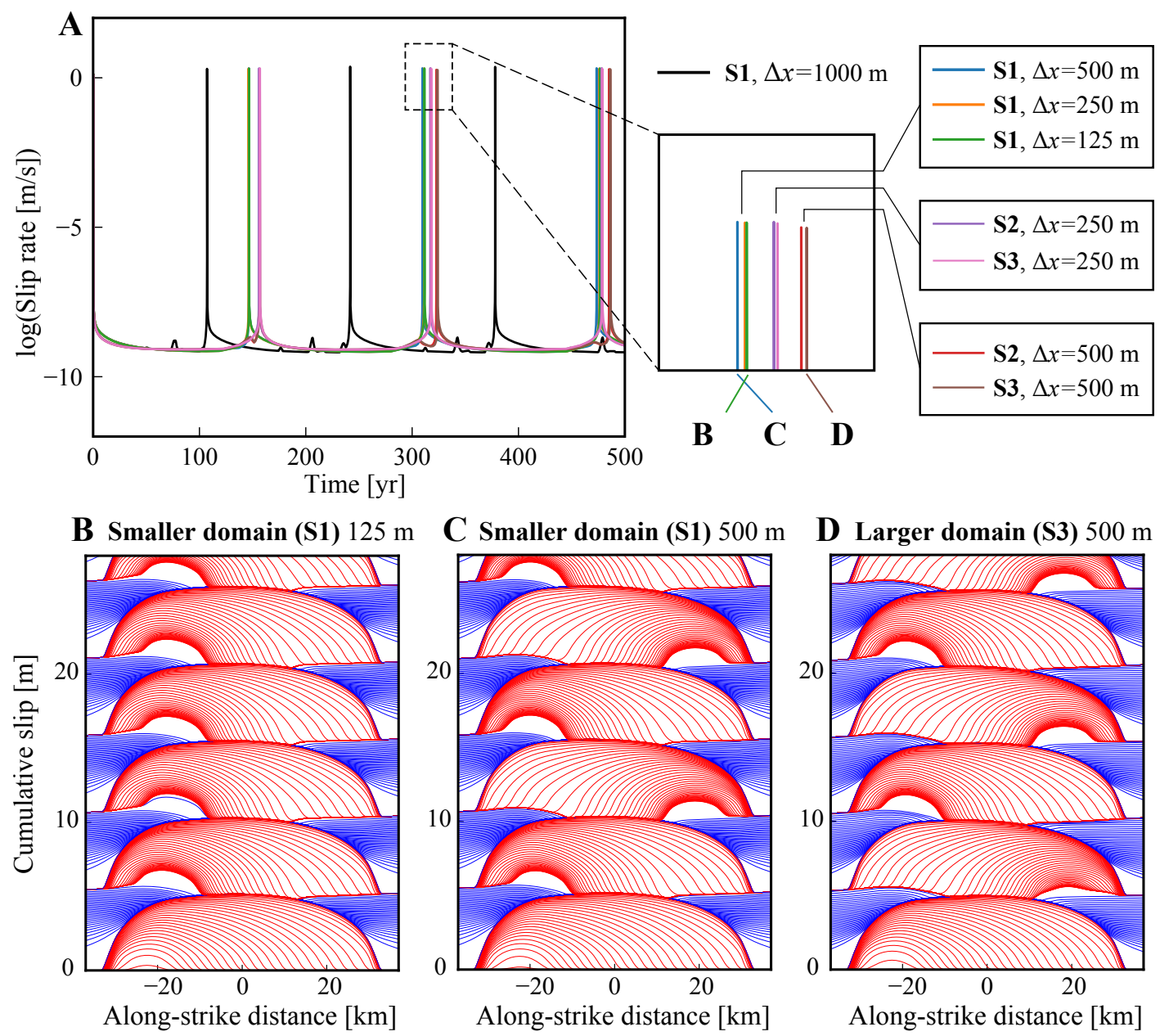

Figure 6. Effect of computational grid spacing and domain size on the self-convergence of SBEM simulations.

(a) Time evolution of maximum slip rates for a suite of BICyclE-2 simulations with different grid spacings

$(\Delta x=125,250,500$, and $1000 \mathrm{~m})$ and domain sizes: $\left(L_{2}, L_{3}\right)=(120 \mathrm{~km}, 90 \mathrm{~km}),(240 \mathrm{~km}, 180 \mathrm{~km})$, or $(480 \mathrm{~km}, 360 \mathrm{~km})$, denoted as S1, S2, or S3, respectively. Cumulative slip in the along-strike direction is plotted every $1 \mathrm{~s}$ for the seismic period (red lines) and every $5 \mathrm{yr}$ for the aseismic period (blue lines) in three simulations with (b) $\Delta x=125 \mathrm{~m}$ and S1; (c) $\Delta x=500 \mathrm{~m}$ and S1; and (d) $\Delta x=500 \mathrm{~m}$ and $\mathrm{S} 3$. The threshold slip rate for the coseismic phase is $V_{\mathrm{th}}=0.01 \mathrm{~m} / \mathrm{s}$. 

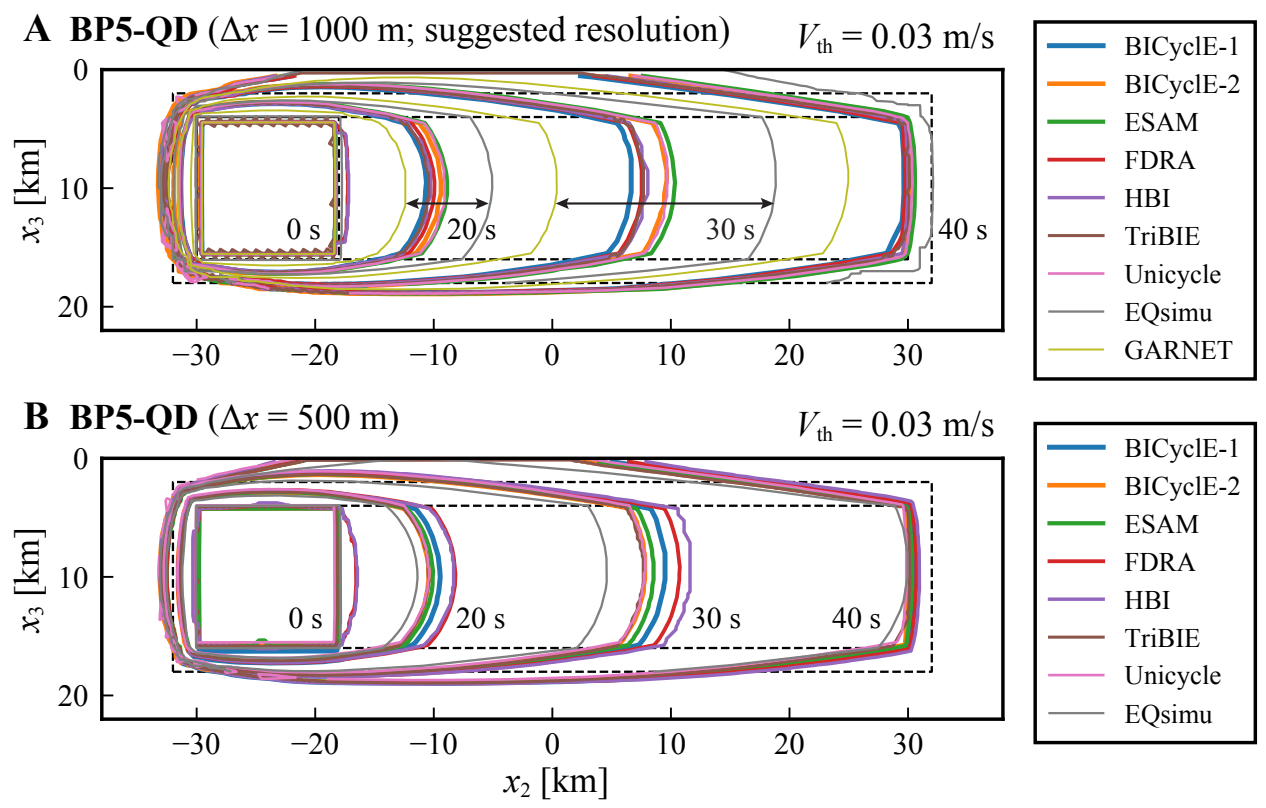

Figure 7. Rupture fronts of the first earthquake in BP5-QD simulations with different numerical resolutions. The contours of rupture fronts indicate $0,20,30$, and $40 \mathrm{~s}$ after the earthquake initiation time in simulations with (a) $\Delta x=1000 \mathrm{~m}$ and (b) $\Delta x=500 \mathrm{~m}$. The threshold slip rate for the coseismic phase, $V_{\mathrm{th}}=0.03 \mathrm{~m} / \mathrm{s}$, is different from that in Figure 4. 

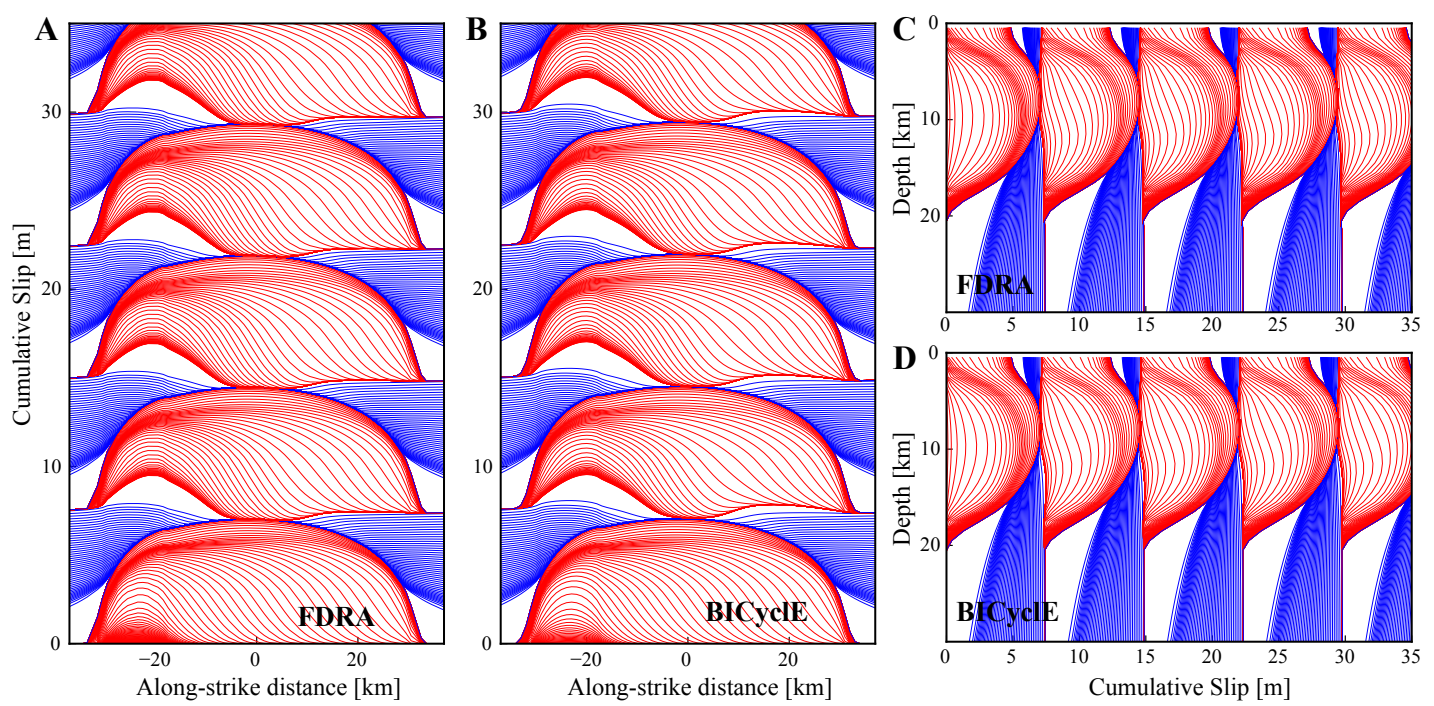

Figure 8. Fault slip evolution in selected BP5-QD simulations. Cumulative fault slip in two simulations $(\Delta x=1000 \mathrm{~m})$ using FDRA and BICyclE is plotted along (a and b) horizontal $\left(x_{3}=10 \mathrm{~km}\right)$ and $(\mathrm{c}$ and $\mathrm{d})$ vertical $\left(x_{2}=0 \mathrm{~km}\right)$ profiles shown in Figure 3. The seismic slip (red lines) is plotted every $1 \mathrm{~s}$ and aseismic slip (blues lines) is plotted every $5 \mathrm{yr}$, with the threshold slip rate $V_{\mathrm{th}}=0.01 \mathrm{~m} / \mathrm{s}$. 


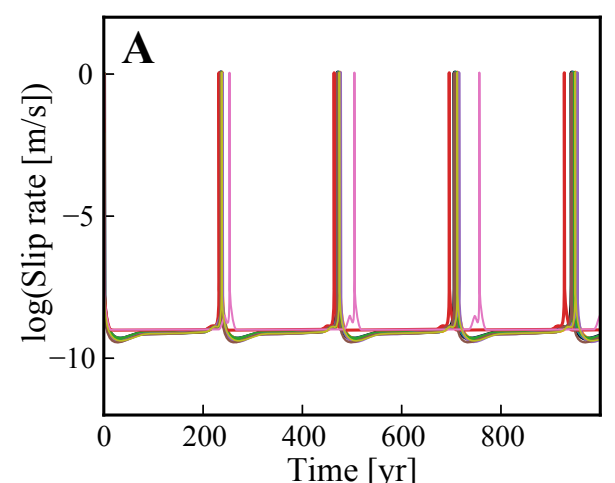

Codes in $\mathbf{A}$
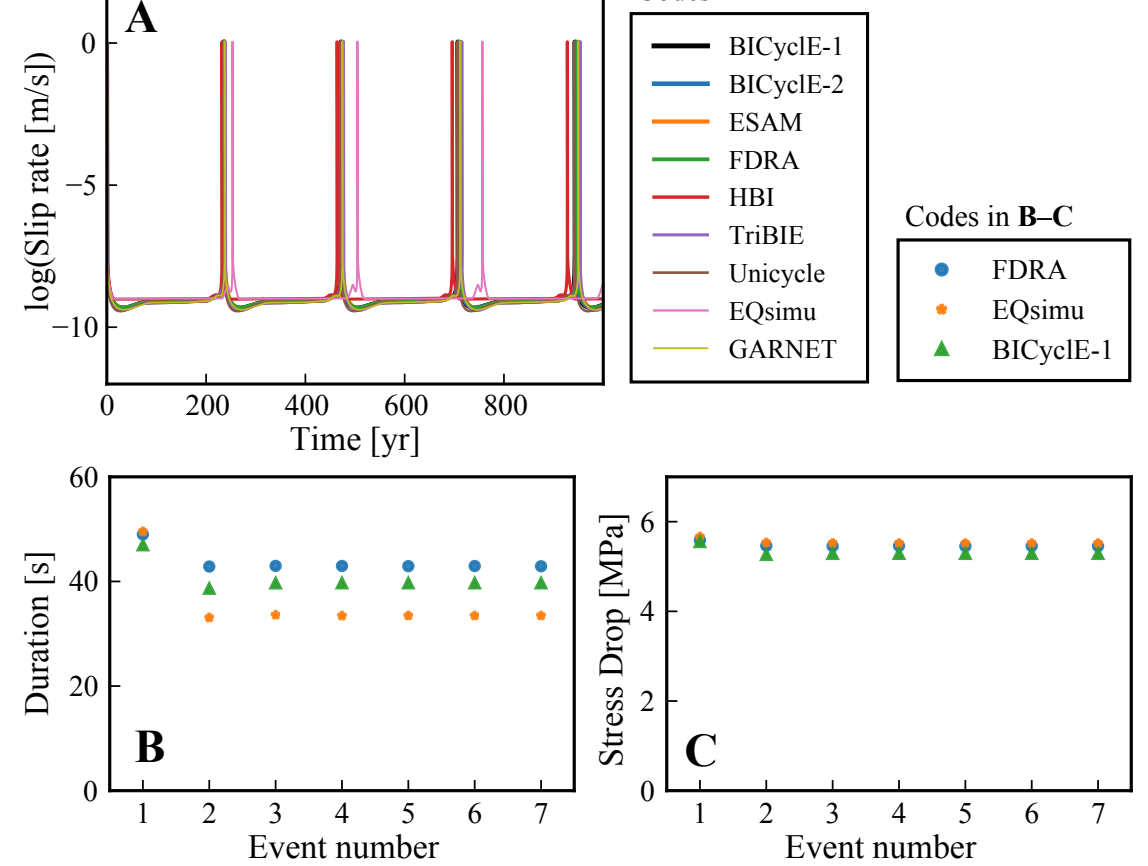

Figure 9. Long-term fault behavior and earthquake characteristics in BP5-QD simulations. (a) Time evolution of maximum slip rates in the seismogenic zone and (b) rupture duration and (c) stress drop for the first seven earthquakes are shown for simulations with $\Delta x=1000 \mathrm{~m}$. 

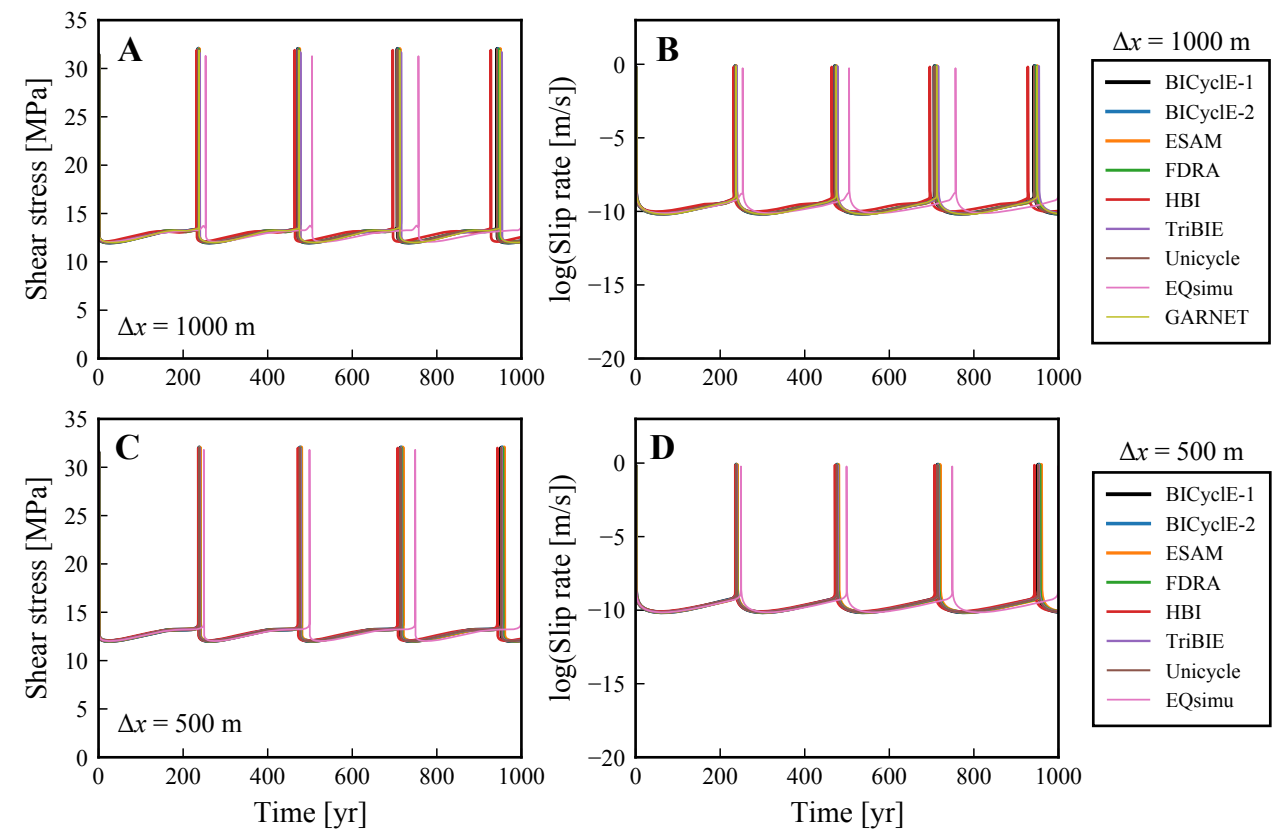

Figure 10. Long-term fault behavior at the surface in BP5-QD simulations. (a and c) Shear stress and (b and d)

slip rates on the fault at the surface $\left(x_{1}=x_{2}=x_{3}=0 \mathrm{~km}\right.$ ) in simulations with (a and b) $\Delta x=1000 \mathrm{~m}$ and (c and d) $\Delta x=500 \mathrm{~m}$. 

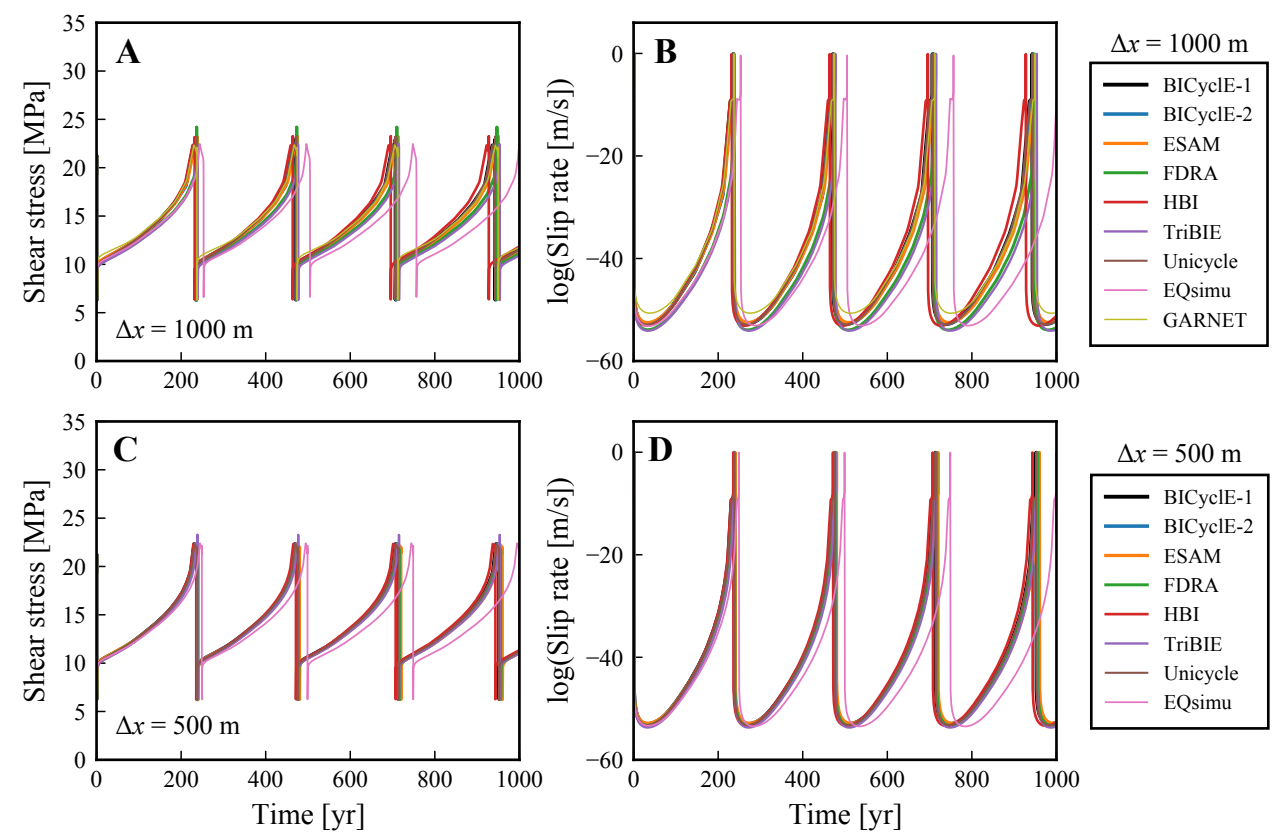

Figure 11. Long-term fault behavior at a seismogenic depth in BP5-QD simulations. (a and c) Shear stress and (b and d) slip rates on the fault at the mid-seismogenic depth $\left(x_{1}=x_{2}=0 \mathrm{~km} ; x_{3}=10 \mathrm{~km}\right)$ in simulations with (a and b) $\Delta x=1000 \mathrm{~m}$ and (c and d) $\Delta x=500 \mathrm{~m}$. Note that the range of vertical axes in panels $\mathrm{b}$ and $\mathrm{d}$ are different from those in Fig. 10. 

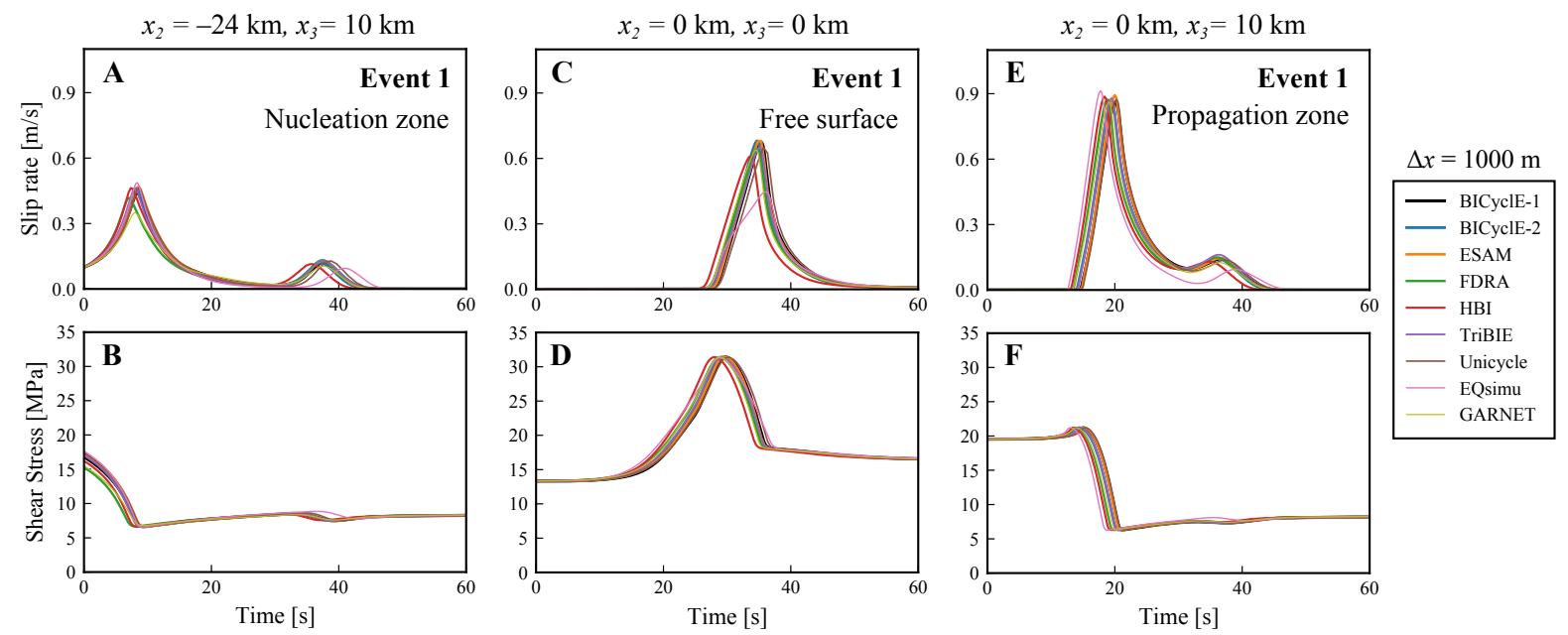

Figure 12. Coseismic rupture of the first event in BP5-QD simulations $(\Delta x=1000 \mathrm{~m})$. Time evolution of (a, $\mathrm{c}$, and e) slip rates and (b, $d$ and f) shear stresses during the first earthquake are shown at different locations on the fault. Panels a and $\mathrm{b}$ refer to a point within the initial nucleation zone $\left(x_{2}=-24 \mathrm{~km} ; x_{3}=10 \mathrm{~km}\right)$. Panels $\mathrm{c}$ and $\mathrm{d}$ refer to a point at the free surface $\left(x_{2}=0 \mathrm{~km} ; x_{3}=0 \mathrm{~km}\right)$. Panels e and $\mathrm{f}$ refer to a point within the propagation zone $\left(x_{2}=0 \mathrm{~km} ; x_{3}=10 \mathrm{~km}\right)$. 

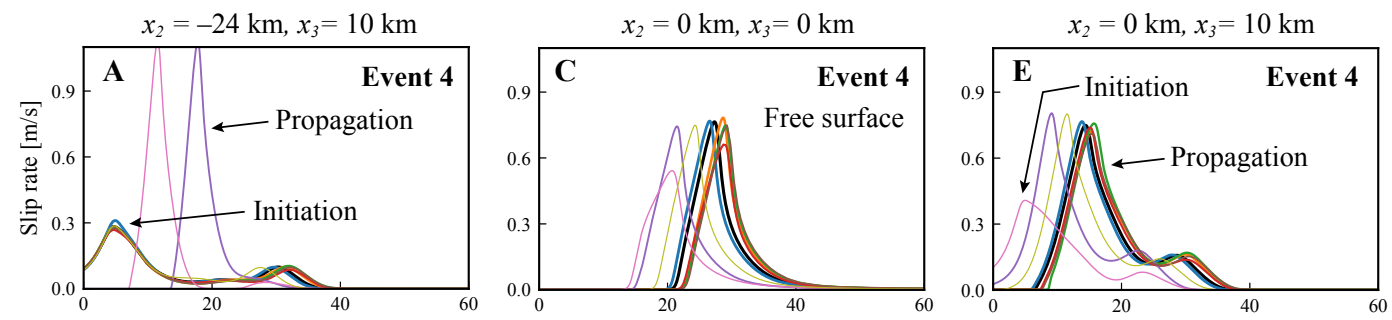

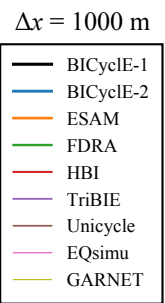
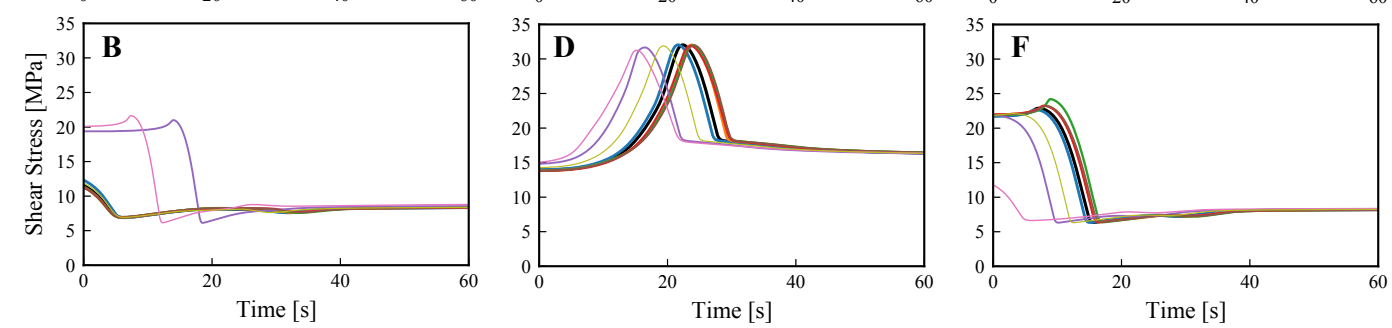

Figure 13. Coseismic rupture of the fourth event in BP5-QD simulations ( $\Delta x=1000 \mathrm{~m})$. Time evolution of (a, c, and e) coseismic slip rates and (b, d and f) shear stresses are shown at the same locations on the fault as in Figure 12. The discrepancy of TriBIE and EQsimu simulations with others are due to different rupture directions. We mark the distinct signals indicating the rupture initiation or propagation in panels a and e. 

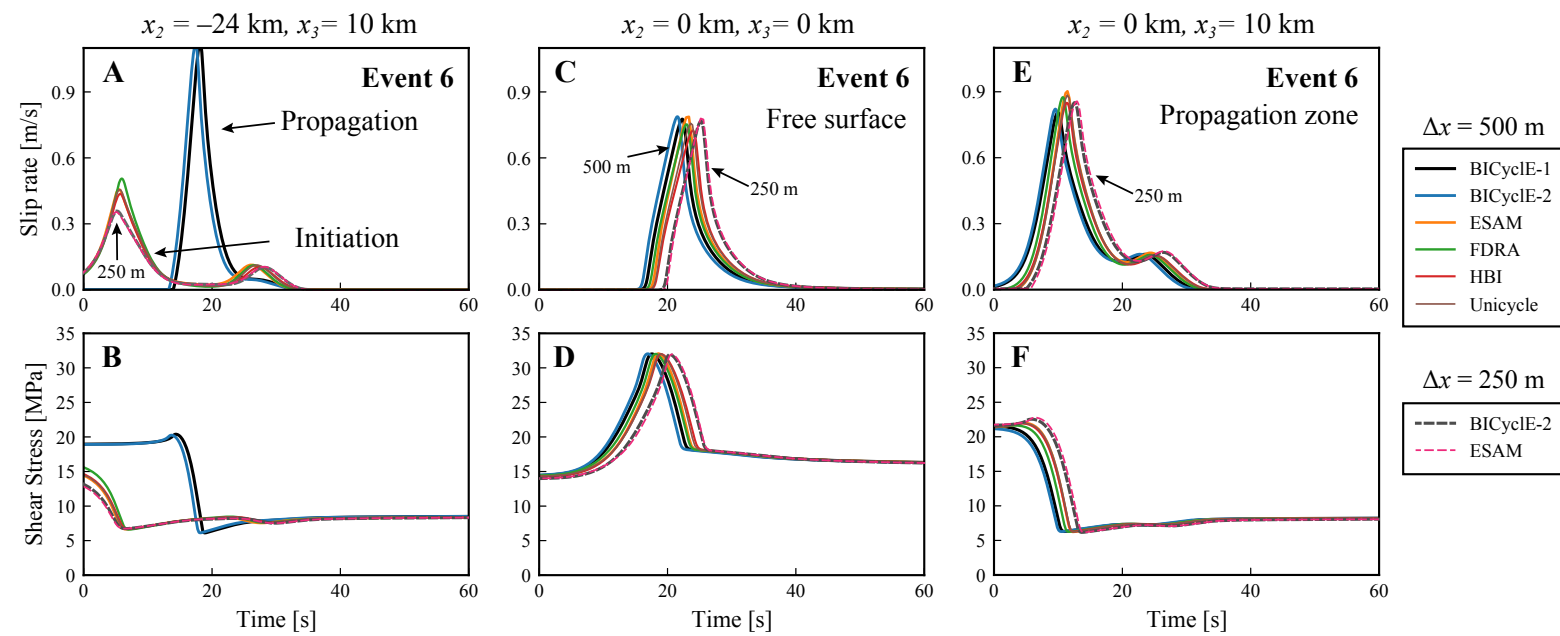

Figure 14. Coseismic rupture of the sixth event in BP5-QD simulations ( $\Delta x=500$ and $250 \mathrm{~m})$. Time evolution of (a, c, and e) coseismic slip rates and (b, d and f) shear stresses are shown at the same locations on the fault as in Figure 12. We mark the distinct signals indicating the rupture initiation or propagation for 500-m simulations, as well as the matching $250-\mathrm{m}$ simulations. 

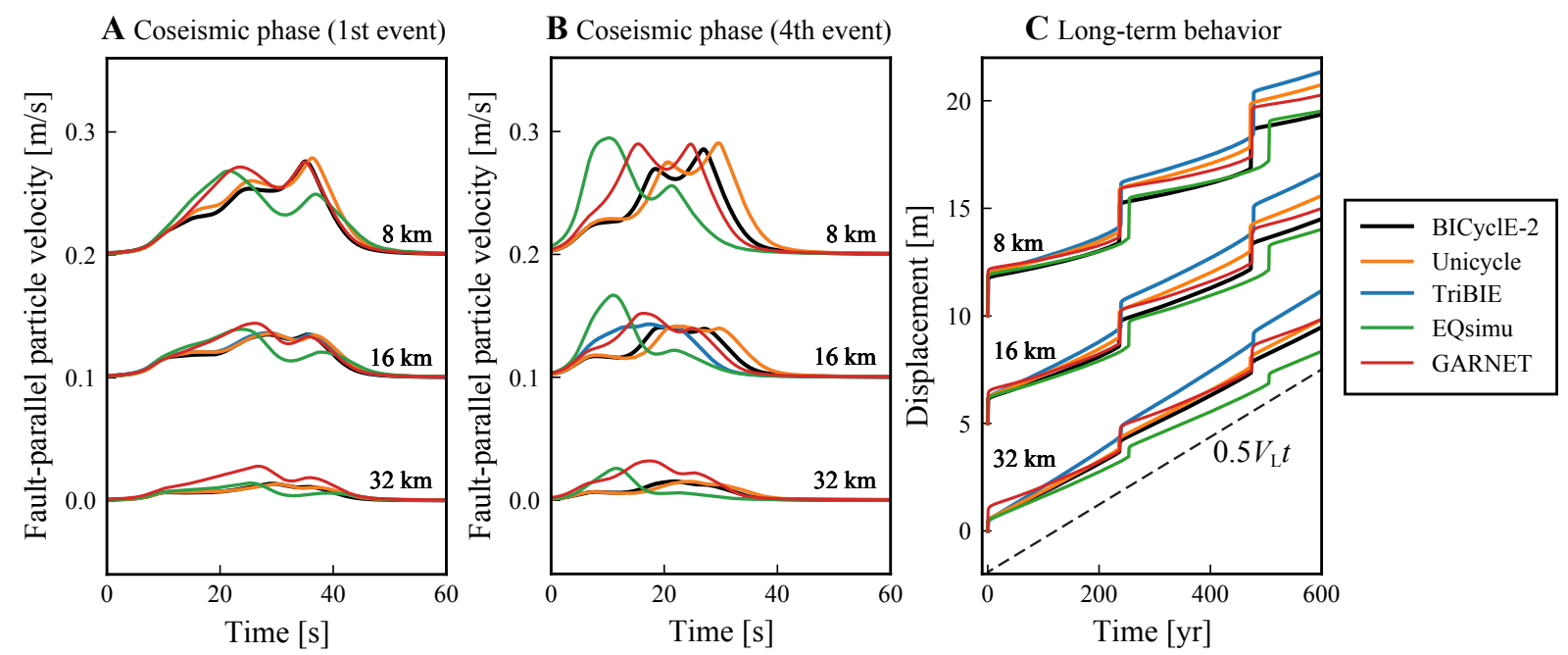

Figure 15. Off-fault ground displacements in BP5-QD simulations. Fault-parallel displacement rates $v_{2}$ during the (a) first and (b) fourth events, and (c) long-term displacement history are shown at three off-fault locations on the surface $\left(x_{1}=8,16\right.$, or $\left.32 \mathrm{~km} ; x_{2}=0 \mathrm{~km} ; x_{3}=0 \mathrm{~km}\right)$. The dashed line indicates the far-field surface displacement $0.5 V_{\mathrm{L}} t$. The time series corresponding to different locations and the dashed line are vertically offset for visualization purpose. 

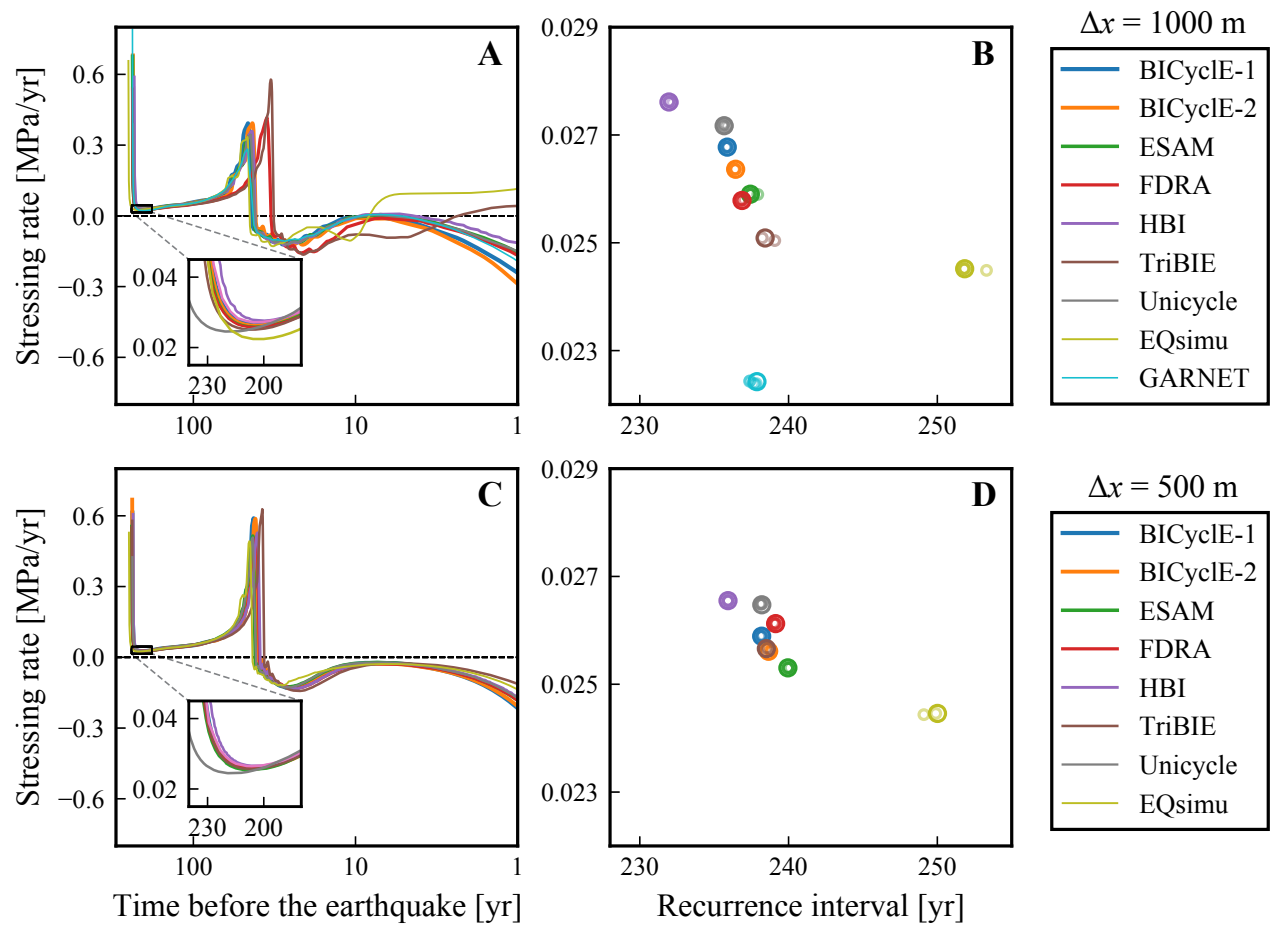

Time before the earthquake $[\mathrm{yr}]$

Recurrence interval [yr]

Figure 16. Interseismic stressing rate history and earthquake recurrence intervals in BP5-QD simulations. (a and c) Stressing rates at the mid-seismogenic depth $\left(x_{2}=0 \mathrm{~km} ; x_{3}=10 \mathrm{~km}\right)$ during the postseismic and interseismic periods before the sixth earthquake. ( $b$ and $d$ ) The minimum interseismic stressing rates (enlarged windows in a and c) and recurrence intervals are shown for the corresponding events (large circles in color) and preceding events (smaller circles in the same color). Simulations with $\Delta x=1000 \mathrm{~m}$ and $\Delta x=500 \mathrm{~m}$ are shown in panels a-b and $\mathrm{c}-\mathrm{d}$, respectively. Due to a shorter simulated time, the fourth event from TriBIE and EQsimu is considered in panels $\mathrm{c}-\mathrm{d}$. 

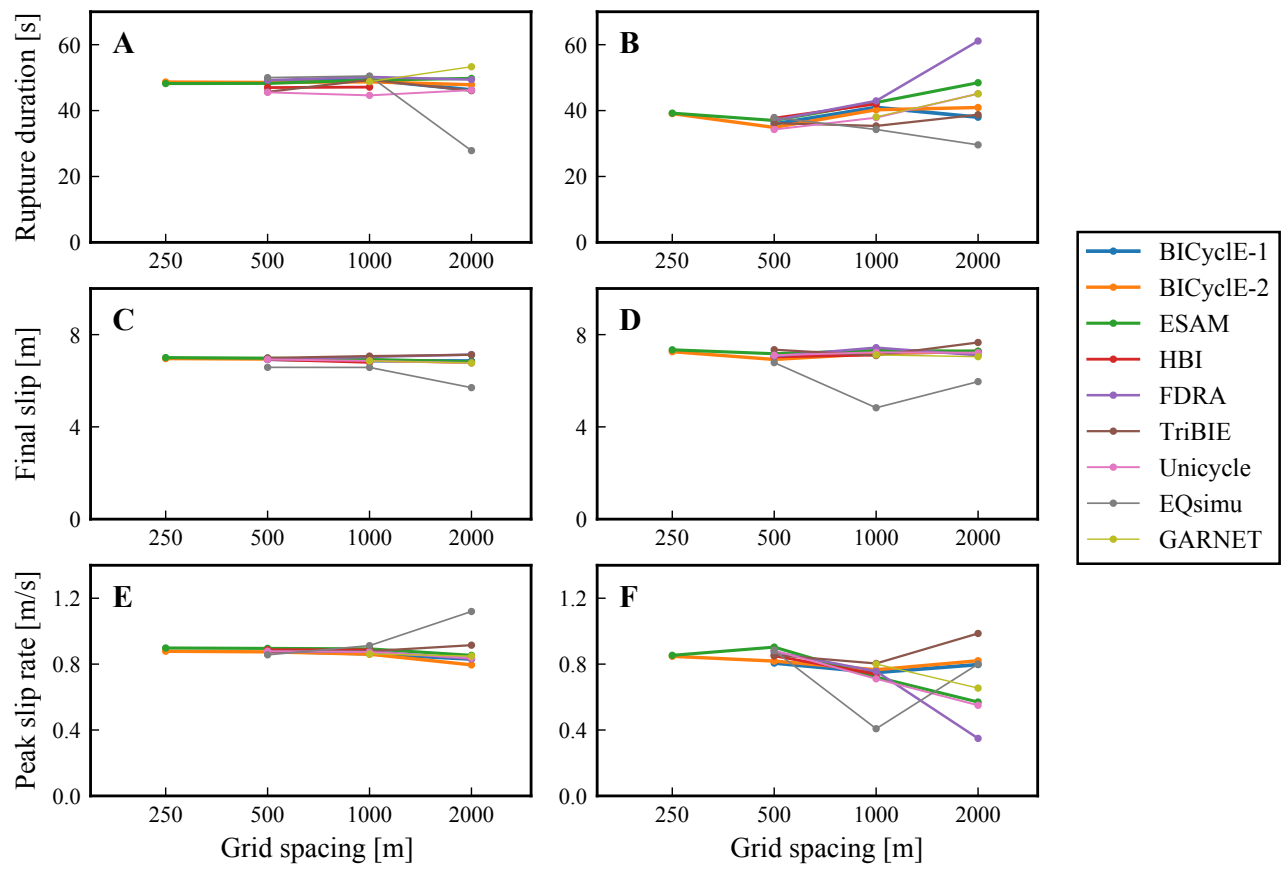

Figure 17. Comparison of earthquake characteristics in simulations with different resolutions. Coseismic rupture durations are shown for the (a) first and (b) sixth events in simulations with $\Delta x=250,500,1000$, and $2000 \mathrm{~m}$, when available. (c and d) Coseismic slip and (e and f) peak slip rate at the mid-seismogenic depth $\left(x_{2}=0 \mathrm{~km} ; x_{3}=10 \mathrm{~km}\right)$ are shown for the (c and e) first and (d and f) sixth event, respectively. Note an exception that the fourth event from TriBIE and EQsimu is considered for $\Delta x=500 \mathrm{~m}$ in panels $\mathrm{b}$, $\mathrm{d}$, and $\mathrm{f}$. Simulation results from each modeling group are plotted as line-connected dots. 
Table 1. Parameters in benchmark problems BP4-QD and BP5-QD

\begin{tabular}{|c|c|c|c|}
\hline Parameter & Symbol & Value in BP4 & Value in BP5 \\
\hline Density & $\rho$ & $2670 \mathrm{~kg} / \mathrm{m}^{3}$ & $2670 \mathrm{~kg} / \mathrm{m}^{3}$ \\
\hline Shear wave speed & $c_{\mathrm{s}}$ & $3.464 \mathrm{~km} / \mathrm{s}$ & $3.464 \mathrm{~km} / \mathrm{s}$ \\
\hline Poisson's ratio & $v$ & 0.25 & 0.25 \\
\hline Effective normal stress & $\sigma_{\mathrm{n}}$ & $50 \mathrm{MPa}$ & $25 \mathrm{MPa}$ \\
\hline Characteristic state evolution distance & $D_{\mathrm{RS}}$ & $0.008 \mathrm{~m}$ & $0.14 \mathrm{~m} / 0.13 \mathrm{~m}^{a}$ \\
\hline Rate-and-state parameter, direct effect, $\mathrm{VW}^{b}$ & $a_{0}$ & 0.0065 & 0.004 \\
\hline Rate-and-state parameter, direct effect, $\mathrm{VS}^{b}$ & $a_{\max }$ & 0.025 & 0.04 \\
\hline Rate-and-state parameter, evolution effect, VW \& VS ${ }^{b}$ & $b_{0}$ & 0.013 & 0.03 \\
\hline Reference slip rate & $V^{*}$ & $10^{-6} \mathrm{~m} / \mathrm{s}$ & $10^{-6} \mathrm{~m} / \mathrm{s}$ \\
\hline Reference coefficient of friction & $f^{*}$ & 0.6 & 0.6 \\
\hline Plate loading rate & $V_{\mathrm{L}}$ & $10^{-9} \mathrm{~m} / \mathrm{s}$ & $10^{-9} \mathrm{~m} / \mathrm{s}$ \\
\hline Initial slip rate & $V_{\text {init }}$ & $10^{-9} \mathrm{~m} / \mathrm{s}$ & $10^{-9} \mathrm{~m} / \mathrm{s}$ \\
\hline Initial slip rate in prescribed nucleation zone & $V_{\mathrm{i}}$ & $0.01 \mathrm{~m} / \mathrm{s}$ & $0.03 \mathrm{~m} / \mathrm{s}$ \\
\hline VW region, (half-) width ${ }^{c}$ & $W$ & $15 \mathrm{~km}$ & $12 \mathrm{~km}$ \\
\hline VW region, length & $L$ & $60 \mathrm{~km}$ & $60 \mathrm{~km}$ \\
\hline VW-VS transition zone, width & $h_{\mathrm{t}}$ & $3 \mathrm{~km}$ & $2 \mathrm{~km}$ \\
\hline Shallow VS region, width & $h_{\mathrm{s}}$ & - & $2 \mathrm{~km}$ \\
\hline Rate-and-state fault, (half-)width ${ }^{c}$ & $W_{\mathrm{f}}$ & $40 \mathrm{~km}$ & $40 \mathrm{~km}$ \\
\hline Rate-and-state fault, length & $L_{\mathrm{f}}$ & $\infty$ & $100 \mathrm{~km}$ \\
\hline Prescribed nucleation zone, width & $w$ & $12 \mathrm{~km}$ & $12 \mathrm{~km}$ \\
\hline Quasi-static process zone size & $\Lambda_{0}$ & $2 \mathrm{~km}$ & $6 \mathrm{~km}$ \\
\hline Nucleation size & $h^{*}$ & $12.4 \mathrm{~km}$ & $12.5 \mathrm{~km}$ \\
\hline Suggested grid spacing & $\Delta x$ & $500 \mathrm{~m}$ & $1000 \mathrm{~m}$ \\
\hline Final simulated time & $t_{\mathrm{f}}$ & 1500 years & 1800 years \\
\hline
\end{tabular}

${ }^{a}$ The value used in the prescribed nucleation zone.

${ }^{b}$ Parameters $a$ and $b$ for velocity-weakening (VW) or velocity-strengthening (VS) regions.

${ }^{c}$ Half-width for BP4-QD and full width for BP5-QD. 
Table 2. Participating SEAS codes and modeling groups

\begin{tabular}{llllll}
\hline Code Name & Type & Simulation $^{a}$ (Group Members) & BP4-QD & BP5-QD & Reference \\
\hline BICyclE & SBEM & jiang (Jiang) & $\checkmark$ & $\checkmark$ & Lapusta and Liu (2009) \\
& & lambert (Lambert, Lapusta) & $\checkmark$ & $\checkmark$ & \\
Motorcycle & SBEM & barbot (Barbot) & $\checkmark$ & & Barbot (2021) \\
ESAM & BEM & liu (Y. Liu) & & $\checkmark$ & Liu and Rice (2007) \\
FDRA & BEM & cattania (Cattania) & & $\checkmark$ & Segall and Bradley (2012) \\
HBI & BEM & ozawa (Ozawa, Ando) & $\checkmark$ & $\checkmark$ & Ozawa et al. (2021) \\
TriBIE & BEM & dli (D. Li) & & $\checkmark$ & Li and Liu (2016) \\
Unicycle & BEM & barbot (Barbot) & $\checkmark$ & $\checkmark$ & Barbot (2019) \\
EQsimu & FEM & dliu (D. Liu, Duan) & $\checkmark$ & $\checkmark$ & Liu et al. (2020) \\
GARNET & FDM & li (M. Li, Dal Zilio, Pranger, & $\checkmark$ & $\checkmark$ & Pranger (2020) \\
& & van Dinther) & & & \\
\hline
\end{tabular}

$a$ The names of simulations displayed on our online platform. 
Table 3. Model parameters in BP4-QD simulations

\begin{tabular}{lllll}
\hline Code Name & Simulation Name & Grid Spacing $(\mathrm{km})^{a}$ & ${\text { Domain Size }(\mathrm{km})^{b}}$ & $\mathrm{BC}^{c}$ \\
\hline BICyclE & jiang & $1,0.5$ & $(192,96, \infty)$ & $\mathrm{P}$ \\
& lambert & $1,0.5,0.25,0.125$ & $(180,90, \infty)$ & $\mathrm{P}$ \\
Motorcycle & barbot & $1,0.5$ & $(120,80, \infty)$ & $\mathrm{P}$ \\
HBI & ozawa & $1,0.5$ & $(120,80, \infty)$ & $\mathrm{D}$ \\
Unicycle & barbot & $1,0.5$ & $(120,80, \infty)$ & $\mathrm{D}$ \\
EQsimu & dliu & 1 & $(120,120,200)$ & $\mathrm{D}$ \\
GARNET & li & 1 & $(120,100,120)$ & $\mathrm{D}$ \\
\hline
\end{tabular}

${ }^{a}$ The grid spacings in simulations submitted by each modeling group.

$b$ The total dimensions of the model domain in the format of $\left(L_{2}, L_{3}, L_{1}\right)$.

${ }^{c}$ Displacement (D) or periodic (P) boundary conditions (BC) in the $x_{2}$ and $x_{3}$ directions. 
Table 4. Model parameters in BP5-QD simulations

\begin{tabular}{lllll}
\hline Code Name & Simulation Name & Grid Spacing $(\mathrm{km})^{a}$ & ${\text { Domain Size }(\mathrm{km})^{a}}$ & $\mathrm{BC}^{a}$ \\
\hline BICyclE & jiang & $2,1,0.5$ & $(192,96, \infty)$ & $\mathrm{P}$ \\
& lambert & $2,1,0.5,0.25$ & $(180,90, \infty)$ & $\mathrm{P}$ \\
ESAM & liu & $2,1,0.5,0.25$ & $(128,40, \infty)$ & ${\mathrm{P} / \mathrm{D}^{b}}^{b}$ \\
FDRA & cattania & $2,1,0.5$ & $\left(10^{4}, 10^{4}, \infty\right)$ & $\mathrm{D}$ \\
HBI & ozawa & $1,0.5$ & $(100,40, \infty)$ & $\mathrm{D}$ \\
TriBIE & dli & $2,1,0.5$ & $(140,60, \infty)$ & $\mathrm{D}$ \\
Unicycle & barbot & $2,1,0.5$ & $(100,40, \infty)$ & $\mathrm{D}$ \\
EQsimu & dliu & $2,1,0.5$ & $(120,60,100)$ & $\mathrm{D}$ \\
GARNET & li & 2,1 & $(120,60,60)$ & $\mathrm{D}$ \\
\hline
\end{tabular}

${ }^{a}$ Same parameters shown in Table 3.

${ }^{b}$ Periodic and displacement BCs in the $x_{2}$ and $x_{3}$ directions, respectively. 


\section{References}

Abdelmeguid, M., X. Ma, and A. Elbanna (2019), A Novel Hybrid Finite Element-Spectral Boundary Integral Scheme for Modeling Earthquake Cycles: Application to Rate and State Faults With Low-Velocity Zones, Journal of Geophysical Research: Solid Earth, 124(12), 12,854-12,881, doi:10/gkr74p.

Allison, K. L., and E. M. Dunham (2018), Earthquake cycle simulations with rate-and-state friction and power-law viscoelasticity, Tectonophysics, 733, 232-256, doi:10.1016/j.tecto.2017.10.021.

Ampuero, J.-P., and A. M. Rubin (2008), Earthquake nucleation on rate and state faults Aging and slip laws, Journal of Geophysical Research: Solid Earth, 113(B1), doi:10.1029/ 2007JB005082.

Andrews, D. J. (1976a), Rupture velocity of plane strain shear cracks, Journal of Geophysical Research (1896-1977), 81(32), 5679-5687, doi:10/b2d43j.

Andrews, D. J. (1976b), Rupture propagation with finite stress in antiplane strain, Journal of Geophysical Research (1896-1977), 81(20), 3575-3582, doi:10/ddkswq.

Barall, M., and R. A. Harris (2015), Metrics for Comparing Dynamic Earthquake Rupture Simulations, Seismological Research Letters, 86(1), 223-235, doi:10/gkr74z.

Barbot, S. (2018), Asthenosphere Flow Modulated by Megathrust Earthquake Cycles, Geophysical Research Letters, 45(12), 6018-6031, doi:10/gdqdqs.

Barbot, S. (2019), Slow-slip, slow earthquakes, period-two cycles, full and partial ruptures, and deterministic chaos in a single asperity fault, Tectonophysics, p. 228171, doi:10/gf629v.

Barbot, S. (2021), A Spectral Boundary-Integral Method for Quasi-Dynamic Ruptures of Multiple Parallel Faults, Bulletin of the Seismological Society of America, 111(3), 1614-1630, doi:10/ gkr75b.

Barbot, S., Y. Fialko, and Y. Bock (2009), Postseismic deformation due to the Mw 6.02004 Parkfield earthquake: Stress-driven creep on a fault with spatially variable rate-and-state friction parameters, Journal of Geophysical Research: Solid Earth, 114(7), B07,405, doi:10.1029/ 2008JB005748.

Barbot, S., N. Lapusta, and J. P. Avouac (2012), Under the hood of the earthquake machine: Toward predictive modeling of the seismic cycle, Science, 336(6082), 707-710, doi:10.1126/ science. 1218796.

Ben-Zion, Y. (2001), Dynamic ruptures in recent models of earthquake faults, Journal of the Mechanics and Physics of Solids, 49(9), 2209-2244, doi:10/c5x4jv.

Ben-Zion, Y., and J. R. Rice (1995), Slip patterns and earthquake populations along different classes of faults in elastic solids, Journal of Geophysical Research: Solid Earth, 100(B7), 12,959-12,983, doi:10.1029/94JB03037. 
Bhat, H. S., R. Dmowska, G. C. P. King, Y. Klinger, and J. R. Rice (2007), Off-fault damage patterns due to supershear ruptures with application to the $2001 \mathrm{Mw} 8.1$ Kokoxili (Kunlun) Tibet earthquake, Journal of Geophysical Research: Solid Earth, 112(6), 1-19, doi:10.1029/ 2006JB004425.

Bizzarri, A., and M. Cocco (2003), Slip-weakening behavior during the propagation of dynamic ruptures obeying rate- and state-dependent friction laws, Journal of Geophysical Research: Solid Earth, 108(B8), doi:10/bcjvcc.

Bizzarri, A., and M. Cocco (2006), A thermal pressurization model for the spontaneous dynamic rupture propagation on a three-dimensional fault: 1. Methodological approach, Journal of Geophysical Research: Solid Earth, 111(B5), doi:10/bd369c.

Bonnet, M. (1999), Boundary Integral Equation Methods for Solids and Fluids | Wiley, Wiley.

Buiter, S. J. H., G. Schreurs, M. Albertz, T. V. Gerya, B. Kaus, W. Landry, L. le Pourhiet, Y. Mishin, D. L. Egholm, M. Cooke, B. Maillot, C. Thieulot, T. Crook, D. May, P. Souloumiac, and C. Beaumont (2016), Benchmarking numerical models of brittle thrust wedges, Journal of Structural Geology, 92, 140-177, doi:10/f9bttc.

Cattania, C., and P. Segall (2019), Crack Models of Repeating Earthquakes Predict Observed Moment-Recurrence Scaling, Journal of Geophysical Research: Solid Earth, 124(1), 476-503, doi:10.1029/2018JB016056.

Cattania, C., and P. Segall (2021), Precursory Slow Slip and Foreshocks on Rough Faults, Journal of Geophysical Research: Solid Earth, 126(4), e2020JB020,430, doi:10/gm4t7n.

Chen, T., and N. Lapusta (2009), Scaling of small repeating earthquakes explained by interaction of seismic and aseismic slip in a rate and state fault model, Journal of Geophysical Research: Solid Earth, 114(1), B01,311, doi:10.1029/2008JB005749.

Dal Zilio, L., Y. van Dinther, T. V. Gerya, and C. C. Pranger (2018), Seismic behaviour of mountain belts controlled by plate convergence rate, Earth and Planetary Science Letters, 482 , 81-92, doi:10/gczxhs.

Dal Zilio, L., N. Lapusta, and J.-P. Avouac (2020), Unraveling Scaling Properties of Slow-Slip Events, Geophysical Research Letters, 47(10), e2020GL087,477, doi:10/gpcbdv.

Dal Zilio, L., N. Lapusta, J.-P. Avouac, and T. Gerya (2022), Subduction earthquake sequences in a non-linear visco-elasto-plastic megathrust, Geophysical Journal International, 229(2), 1098-1121, doi:10/gn99ms.

Das, S., and K. Aki (1977), Fault plane with barriers: A versatile earthquake model, Journal of Geophysical Research (1896-1977), 82(36), 5658-5670, doi:10/cj7wwm.

Day, S. M. (1982), Three-dimensional simulation of spontaneous rupture: The effect of nonuniform prestress, Bulletin of the Seismological Society of America, 72(6), 1881-1902. 
Day, S. M., L. A. Dalguer, N. Lapusta, and Y. Liu (2005), Comparison of finite difference and boundary integral solutions to three-dimensional spontaneous rupture, Journal of Geophysical Research: Solid Earth, 110(B12), doi:10.1029/2005JB003813.

Dieterich, J. H. (1979), Modeling of rock friction 1. Experimental results and constitutive equations, Journal of Geophysical Research, [Solid Earth], 84(B5), 2161-2168, doi:10.1029/ JB084iB05p02161.

Dieterich, J. H., K. B. Richards-Dinger, and K. A. Kroll (2015), Modeling Injection-Induced Seismicity with the Physics-Based Earthquake Simulator RSQSim, Seismological Research Letters, 86(4), 1102-1109, doi:10/gg8hjn.

Duan, B., and S. M. Day (2008), Inelastic strain distribution and seismic radiation from rupture of a fault kink, Journal of Geophysical Research: Solid Earth, 113(B12), doi:10/fthjcb.

Dublanchet, P. (2018), The dynamics of earthquake precursors controlled by effective friction, Geophysical Journal International, 212(2), 853-871, doi:10/gcvdnw.

Dublanchet, P., P. Bernard, and P. Favreau (2013), Creep modulation of Omori law generated by a Coulomb stress perturbation in a 3-D rate-and-state asperity model, Journal of Geophysical Research E: Planets, 118(9), 4774-4793, doi:10.1002/jgrb.50311.

Dunham, E. M., D. Belanger, L. Cong, and J. E. Kozdon (2011a), Earthquake Ruptures with Strongly Rate-Weakening Friction and Off-Fault Plasticity, Part 2: Nonplanar Faults, Bulletin of the Seismological Society of America, 101(5), 2308-2322, doi:10/bnsr54.

Dunham, E. M., D. Belanger, L. Cong, and J. E. Kozdon (2011b), Earthquake Ruptures with Strongly Rate-Weakening Friction and Off-Fault Plasticity, Part 1: Planar Faults, Bulletin of the Seismological Society of America, 101(5), 2296-2307, doi:10/d7t5n4.

Erickson, B. A., and E. M. Dunham (2014), An efficient numerical method for earthquake cycles in heterogeneous media: Alternating subbasin and surface-rupturing events on faults crossing a sedimentary basin, Journal of Geophysical Research: Solid Earth, 119(4), 3290-3316, doi:10/ gkr74x.

Erickson, B. A., E. M. Dunham, and A. Khosravifar (2017), A finite difference method for off-fault plasticity throughout the earthquake cycle, Journal of the Mechanics and Physics of Solids, 109, 50-77, doi:10/gcg5ft.

Erickson, B. A., J. Jiang, M. Barall, N. Lapusta, E. M. Dunham, R. Harris, L. S. Abrahams, K. L. Allison, J.-P. Ampuero, S. Barbot, C. Cattania, A. Elbanna, Y. Fialko, B. Idini, J. E. Kozdon, V. Lambert, Y. Liu, Y. Luo, X. Ma, M. B. McKay, P. Segall, P. Shi, M. van den Ende, and M. Wei (2020), The Community Code Verification Exercise for Simulating Sequences of Earthquakes and Aseismic Slip (SEAS), Seismological Research Letters, 91(2A), 874-890, doi:10.1785/0220190248. 
Floyd, M. A., R. J. Walters, J. R. Elliott, G. J. Funning, J. L. Svarc, J. R. Murray, A. J. Hooper, Y. Larsen, P. Marinkovic, R. Bürgmann, I. A. Johanson, and T. J. Wright (2016), Spatial variations in fault friction related to lithology from rupture and afterslip of the 2014 South Napa, California, earthquake, Geophysical Research Letters, 43(13), 6808-6816, doi:10/f82v2r.

Freund, L. (1990), Dynamic Fracture Mechanics, Cambridge Monographs on Mechanics, Cambridge University Press.

Gabriel, A. A., J. P. Ampuero, L. A. Dalguer, and P. M. Mai (2012), The transition of dynamic rupture styles in elastic media under velocity-weakening friction, Journal of Geophysical Research, [Solid Earth], 117(9), B09,311, doi:10.1029/2012JB009468.

Galis, M., C. Pelties, J. Kristek, P. Moczo, J.-P. Ampuero, and P. M. Mai (2015), On the initiation of sustained slip-weakening ruptures by localized stresses, Geophysical Journal International, 200(2), 890-909, doi:10/f63pm9.

Harris, R. A., R. J. Archuleta, and S. M. Day (1991), Fault steps and the dynamic rupture process: 2-D numerical simulations of a spontaneously propagating shear fracture, Geophysical Research Letters, 18(5), 893-896, doi:10/c7hjgf.

Harris, R. A., M. Barall, R. Archuleta, E. Dunham, B. Aagaard, J. P. Ampuero, H. Bhat, V. Cruz-Atienza, L. Dalguer, P. Dawson, S. Day, B. Duan, G. Ely, Y. Kaneko, Y. Kase, N. Lapusta, Y. Liu, S. Ma, D. Oglesby, K. Olsen, A. Pitarka, S. Song, and E. Templeton (2009), The SCEC/USGS Dynamic Earthquake Rupture Code Verification Exercise, Seismological Research Letters, 80(1), 119-126, doi:10.1785/gssrl.80.1.119.

Harris, R. A., M. Barall, B. Aagaard, S. Ma, D. Roten, K. Olsen, B. Duan, D. Liu, B. Luo, K. Bai, J.-P. Ampuero, Y. Kaneko, A.-A. Gabriel, K. Duru, T. Ulrich, S. Wollherr, Z. Shi, E. Dunham, S. Bydlon, Z. Zhang, X. Chen, S. N. Somala, C. Pelties, J. Tago, V. M. Cruz-Atienza, J. Kozdon, E. Daub, K. Aslam, Y. Kase, K. Withers, and L. Dalguer (2018), A Suite of Exercises for Verifying Dynamic Earthquake Rupture Codes, Seismological Research Letters, 89(3), 1146-1162, doi:10/gd3pzn.

Harris, R. A., M. Barall, D. A. Lockner, D. E. Moore, D. A. Ponce, R. W. Graymer, G. Funning, C. A. Morrow, C. Kyriakopoulos, and D. Eberhart-Phillips (2021), A Geology and Geodesy Based Model of Dynamic Earthquake Rupture on the Rodgers Creek-Hayward-Calaveras Fault System, California, Journal of Geophysical Research: Solid Earth, 126(3), e2020JB020,577, doi:10/gn4ck4.

Hawthorne, J. C., and A. M. Rubin (2013), Laterally propagating slow slip events in a rate and state friction model with a velocity-weakening to velocity-strengthening transition, Journal of Geophysical Research: Solid Earth, 118(7), 3785-3808, doi:10/gpcbjd.

Herrendörfer, R., T. Gerya, and Y. van Dinther (2018), An Invariant Rate- and State-Dependent Friction Formulation for Viscoeastoplastic Earthquake Cycle Simulations, Journal of Geophysical Research: Solid Earth, 123(6), 5018-5051, doi:10/gd76br. 
Hori, T., N. Kato, K. Hirahara, T. Baba, and Y. Kaneda (2004), A numerical simulation of earthquake cycles along the Nankai Trough in southwest Japan: Lateral variation in frictional property due to the slab geometry controls the nucleation position, Earth and planetary science letters, 228(3), 215-226, doi:10.1016/j.eps1.2004.09.033.

Jiang, J., and Y. Fialko (2016), Reconciling seismicity and geodetic locking depths on the Anza section of the San Jacinto fault, Geophysical research letters, 43(20), 10,663-10,671, doi:10. 1002/2016GL071113.

Jiang, J., and N. Lapusta (2016), Deeper penetration of large earthquakes on seismically quiescent faults, Science, 352(6291), 1293-1297, doi:10.1126/science.aaf1496.

Jiang, J., and N. Lapusta (2017), Connecting depth limits of interseismic locking, microseismicity, and large earthquakes in models of long-term fault slip, Journal of Geophysical Research: Solid Earth, 122(8), 6491-6523, doi:10.1002/2017JB014030.

Johnson, K. M., R. Bürgmann, and K. Larson (2006), Frictional properties on the San Andreas fault near Parkfield, California, inferred from models of afterslip following the 2004 earthquake, Bulletin of the Seismological Society of America, 96(4 B), S321-S338, doi:10.1785/0120050808.

Kaneko, Y., and N. Lapusta (2008), Variability of earthquake nucleation in continuum models of rate-and-state faults and implications for aftershock rates, Journal of Geophysical Research, [Solid Earth], 113(12), B12,312, doi:10.1029/2007JB005154.

Kaneko, Y., J. P. Ampuero, and N. Lapusta (2011), Spectral-element simulations of long-term fault slip: Effect of low-rigidity layers on earthquake-cycle dynamics, Journal of Geophysical Research, [Solid Earth], 116(10), 1-18, doi:10.1029/2011JB008395.

Kato, N. (2016), Earthquake Cycles in a Model of Interacting Fault Patches: Complex Behavior at Transition from Seismic to Aseismic Slip, Bulletin of the Seismological Society of America, 106(4), 1772-1787, doi:10/f8wkfz.

Kozdon, J. E., and E. M. Dunham (2013), Rupture to the Trench: Dynamic rupture simulations of the 11 march 2011 Tohoku earthquake, Bulletin of the Seismological Society of America, 103(2 B), 1275-1289, doi:10.1785/0120120136.

Kroll, K. A., and E. S. Cochran (2021), Stress Controls Rupture Extent and Maximum Magnitude of Induced Earthquakes, Geophysical Research Letters, 48(11), e2020GL092,148, doi:10/gj5hcf.

Lambert, V., and S. Barbot (2016), Contribution of viscoelastic flow in earthquake cycles within the lithosphere-asthenosphere system, Geophysical research letters, 43(19), 10,142-10,154, doi:10.1002/2016GL070345.

Lambert, V., and N. Lapusta (2021), Resolving Simulated Sequences of Earthquakes and Fault Interactions: Implications for Physics-Based Seismic Hazard Assessment, Journal of Geophysical Research: Solid Earth, 126(10), e2021JB022,193, doi:10/gnt7gk. 
Lapusta, N., and Y. Liu (2009), Three-dimensional boundary integral modeling of spontaneous earthquake sequences and aseismic slip, Journal of Geophysical Research, [Solid Earth], 114(9), doi:10.1029/2008JB005934.

Lapusta, N., and J. R. Rice (2003), Low-heat and low-stress fault operation in earthquake models of statically strong but dynamically weak faults, American Geophysical Union, Fall Meeting 2003, abstract id. S51B-02, p. B2.

Lapusta, N., J. R. Rice, Y. Ben-Zion, and G. Zheng (2000), Elastodynamic analysis for slow tectonic loading with spontaneous rupture episodes on faults with rate- and state-dependent friction, Journal of Geophysical Research, [Solid Earth], 105(B10), 23,765-23,789, doi:10. 1029/2000JB900250.

Li, D., and Y. Liu (2016), Spatiotemporal evolution of slow slip events in a nonplanar fault model for northern Cascadia subduction zone, Journal of Geophysical Research: Solid Earth, 121(9), 6828-6845, doi:10/f88d7f.

Li, D., and Y. Liu (2017), Modeling slow-slip segmentation in Cascadia subduction zone constrained by tremor locations and gravity anomalies, Journal of Geophysical Research: Solid Earth, 122(4), 3138-3157, doi:10/f99bkr.

Liu, D., B. Duan, and B. Luo (2020), EQsimu: A 3-D finite element dynamic earthquake simulator for multicycle dynamics of geometrically complex faults governed by rate- and state-dependent friction, Geophysical Journal International, 220(1), 598-609, doi:10/gj263v.

Liu, Y. (2013), Numerical simulations on megathrust rupture stabilized under strong dilatancy strengthening in slow slip region, Geophysical Research Letters, 40(7), 1311-1316, doi:10/ gkr74w.

Liu, Y., and J. R. Rice (2005), Aseismic slip transients emerge spontaneously in three-dimensional rate and state modeling of subduction earthquake sequences, Journal of Geophysical Research, [Solid Earth], 110(8), 1-14, doi:10.1029/2004JB003424.

Liu, Y., and J. R. Rice (2007), Spontaneous and triggered aseismic deformation transients in a subduction fault model, Journal of Geophysical Research, [Solid Earth], 112(9), doi:10.1029/ 2007JB004930.

Lozos, J. C., D. D. Oglesby, B. Duan, and S. G. Wesnousky (2011), The Effects of Double Fault Bends on Rupture Propagation: A Geometrical Parameter Study, Bulletin of the Seismological Society of America, 101(1), 385-398, doi:10/bcr7j4.

Luo, B., B. Duan, and D. Liu (2020), 3D Finite-Element Modeling of Dynamic Rupture and Aseismic Slip over Earthquake Cycles on Geometrically Complex Faults, Bulletin of the Seismological Society of America, 110(6), 2619-2637, doi:10/ghnnc7.

Luo, Y., and J.-P. Ampuero (2018), Stability of faults with heterogeneous friction properties and effective normal stress, Tectonophysics, 733, 257-272, doi:10/gdn68r. 
Luo, Y., J. P. Ampuero, P. Galvez, M. van den Ende, and B. Idini (2017), QDYN: A Quasi-DYNamic Earthquake Simulator (v1.1), doi:10.5281/zenodo.322459.

Ma, S., and G. C. Beroza (2008), Rupture dynamics on a bimaterial interface for dipping faults, Bulletin of the Seismological Society of America, 98(4), 1642-1658, doi:10.1785/0120070201.

Madariaga, R., K. Olsen, and R. Archuleta (1998), Modeling dynamic rupture in a 3D earthquake fault model, Bulletin of the Seismological Society of America, 88(5), 1182-1197, doi:10.1109/ LPT.2005.859990.

Marone, C. (1998), Laboratory-derived friction laws and their application to seismic faulting, Аnпи. Rev. Earth Planet. Sci., 26(1), 643-696, doi:10/d9shmt.

Matsui, H., E. Heien, J. Aubert, J. M. Aurnou, M. Avery, B. Brown, B. A. Buffett, F. Busse, U. R. Christensen, C. J. Davies, N. Featherstone, T. Gastine, G. A. Glatzmaier, D. Gubbins, J.-L. Guermond, Y.-Y. Hayashi, R. Hollerbach, L. J. Hwang, A. Jackson, C. A. Jones, W. Jiang, L. H. Kellogg, W. Kuang, M. Landeau, P. Marti, P. Olson, A. Ribeiro, Y. Sasaki, N. Schaeffer, R. D. Simitev, A. Sheyko, L. Silva, S. Stanley, F. Takahashi, S.-i. Takehiro, J. Wicht, and A. P. Willis (2016), Performance benchmarks for a next generation numerical dynamo model, Geochemistry, Geophysics, Geosystems, 17(5), 1586-1607, doi:10/f8vkhm.

Maxwell, R. M., M. Putti, S. Meyerhoff, J.-O. Delfs, I. M. Ferguson, V. Ivanov, J. Kim, O. Kolditz, S. J. Kollet, M. Kumar, S. Lopez, J. Niu, C. Paniconi, Y.-J. Park, M. S. Phanikumar, C. Shen, E. A. Sudicky, and M. Sulis (2014), Surface-subsurface model intercomparison: A first set of benchmark results to diagnose integrated hydrology and feedbacks, Water Resources Research, 50(2), 1531-1549, doi:10/f5zqnx.

McClure, M. W., and R. N. Horne (2011), Investigation of injection-induced seismicity using a coupled fluid flow and rate/state friction model, GEOPHYSICS, 76(6), WC181-WC198, doi:10/ fzj6qw.

Mckay, M. B., B. A. Erickson, and J. E. Kozdon (2019), A computational method for earthquake cycles within anisotropic media, Geophysical Journal International, 219(2), 816-833, doi:10/ gkr74j.

Mele Veedu, M., and S. Barbot (2016), The Parkfield tremors reveal slow and fast ruptures on the same asperity, Nature, 532(7599), 361-365, doi:10/f8j8rw.

Michel, S., J.-P. Avouac, N. Lapusta, and J. Jiang (2017), Pulse-like partial ruptures and high-frequency radiation at creeping-locked transition during megathrust earthquakes, Geophysical research letters, 44(16), 8345-8351, doi:10.1002/2017GL074725.

Mikumo, T., and T. Miyatake (1978), Dynamical rupture process on a three-dimensional fault with non-uniform frictions and near-field seismic waves, Geophysical Journal International, 54(2), 417-438, doi:10/bc54cf. 
Mikumo, T., and T. Miyatake (1993), Dynamic Rupture Processes on a Dipping Fault, and Estimates of Stress Drop and Strength Excess from the Results of Waveform Inversion, Geophysical Journal International, 112(3), 481-496, doi:10/dx9nns.

Mitsui, Y., and Y. Iio (2011), How did the 2011 off the Pacific coast of Tohoku Earthquake start and grow? The role of a conditionally stable area, Earth, Planets and Space, 63(7), 755-759, doi:10.5047/eps.2011.05.007.

Nakata, R., M. Hyodo, and T. Hori (2012), Numerical simulation of afterslips and slow slip events that occurred in the same area in Hyuga-nada of southwest Japan: Simulation of afterslips and slow slip events, Geophysical Journal International, 190(2), 1213-1220, doi:10/f344rw.

Nearing, G. S., B. L. Ruddell, M. P. Clark, B. Nijssen, and C. Peters-Lidard (2018), Benchmarking and Process Diagnostics of Land Models, Journal of Hydrometeorology, 19(11), 1835-1852, doi:10/gfqkkr.

Nielsen, S. B., J. M. Carlson, and K. B. Olsen (2000), Influence of friction and fault geometry on earthquake rupture, Journal of Geophysical Research: Solid Earth, 105(B3), 6069-6088, doi:10/ c4z6zg.

Noda, H., and N. Lapusta (2010), Three-dimensional earthquake sequence simulations with evolving temperature and pore pressure due to shear heating: Effect of heterogeneous hydraulic diffusivity, Journal of Geophysical Research, [Solid Earth], 115(12), doi:10.1029/ 2010JB007780.

Noda, H., M. Nakatani, and T. Hori (2013), A slower fault may produce a smaller preseismic moment rate: Non-1/tfacceleration of moment rate during nucleation and dependency on the background slip rate, Geophysical research letters, 40(18), 4850-4854, doi:10.1002/grl.50962.

Okada, Y. (1992), Internal deformation due to shear and tensile faults in a half-space, Bulletin of the Seismological Society of America, 82(2), 1018-1040.

Olsen, K. B., R. Madariaga, and R. J. Archuleta (1997), Three-Dimensional Dynamic Simulation of the 1992 Landers Earthquake, Science, 278(5339), 834-838, doi:10/cwz9hp.

Ozawa, S., A. Ida, T. Hoshino, and R. Ando (2021), Large-scale earthquake sequence simulations of 3D geometrically complex faults using the boundary element method accelerated by lattice H-matrices on distributed memory computer systems, arXiv:2110.12165.

Palmer, A. C., and J. R. Rice (1973), The Growth of Slip Surfaces in the Progressive Failure of Over-Consolidated Clay, Proceedings of the Royal Society A: Mathematical, Physical and Engineering Sciences, 332(1591), 527-548, doi:10.1098/rspa.1973.0040.

Perfettini, H., and J. P. Avouac (2007), Modeling afterslip and aftershocks following the 1992 Landers earthquake, Journal of Geophysical Research, [Solid Earth], 112(7), B07,409, doi:10. 1029/2006JB004399. 
Pranger, C. C. (2020), Unstable physical processes operating on self-governing fault systems, Improved Modeling Methodology, Doctoral Thesis, ETH Zurich, doi:10.3929/ ethz-b-000475293.

Qiu, Q., E. M. Hill, S. Barbot, J. Hubbard, W. Feng, E. O. Lindsey, L. Feng, K. Dai, S. V. Samsonov, and P. Tapponnier (2016), The mechanism of partial rupture of a locked megathrust: The role of fault morphology, Geology, 44(10), 875-878, doi:10/f85mdp.

Rice, J. R. (1993), Spatio-temporal complexity of slip on a fault, Journal of Geophysical Research: Solid Earth, 98(B6), 9885-9907, doi:10/c9gs59.

Rice, J. R., and A. L. Ruina (1983), Stability of Steady Frictional Slipping, Journal of Applied Mechanics, 50(2), 343, doi:10.1115/1.3167042.

Rice, J. R., and S. T. Tse (1986), Dynamic motion of a single degree of freedom system following a rate and state dependent friction law, Journal of Geophysical Research: Solid Earth, 91(B1), 521-530, doi:10/fgjzmm.

Richards-Dinger, K., and J. H. Dieterich (2012), RSQSim Earthquake Simulator, Seismological Research Letters, 83(6), 983-990, doi:10.1785/0220120105.

Ripperger, J., J. P. Ampuero, P. M. Mai, and D. Giardini (2007), Earthquake source characteristics from dynamic rupture with constrained stochastic fault stress, Journal of Geophysical Research, [Solid Earth], 112(4), doi:10.1029/2006JB004515.

Robinson, R., and R. Benites (1995), Synthetic seismicity models of multiple interacting faults, Journal of Geophysical Research: Solid Earth, 100(B9), 18,229-18,238, doi:10/b3wvkw.

Robinson, R., and R. Benites (1996), Synthetic seismicity models for the Wellington Region, New Zealand: Implications for the temporal distribution of large events, Journal of Geophysical Research: Solid Earth, 101(B12), 27,833-27,844, doi:10/d5mzmh.

Robinson, R., and R. Benites (2001), Upgrading a synthetic seismicity model for more realistic fault ruptures, Geophysical Research Letters, 28(9), 1843-1846, doi:10/crm89p.

Romanet, P., and S. Ozawa (2021), Fully Dynamic Earthquake Cycle Simulations on a Nonplanar Fault Using the Spectral Boundary Integral Element Method (sBIEM), Bulletin of the Seismological Society of America, doi:10/gn8sjc.

Rubin, A. M., and J. P. Ampuero (2005), Earthquake nucleation on (aging) rate and state faults, Journal of Geophysical Research, [Solid Earth], 110(11), 1-24, doi:10.1029/2005JB003686.

Ruina, A. (1983), Slip instability and state variable friction laws, Journal of geophysical research, 88(B12), 10,359-10,370, doi:10.1029/JB088iB12p10359.

Sathiakumar, S., S. Barbot, and J. Hubbard (2020), Earthquake Cycles in Fault-Bend Folds, Journal of Geophysical Research: Solid Earth, 125(8), e2019JB018,557, doi:10/gm4t7s.

Savage, J. C., and R. O. Burford (1973), Geodetic determination of relative plate motion in central California, Journal of geophysical research, 78(5), 832-845, doi:10.1029/JB078i005p00832. 
Segall, P., and A. M. Bradley (2012), The Role of Thermal Pressurization and Dilatancy in Controlling the Rate of Fault Slip, Journal of Applied Mechanics, 79(3), 031,013, doi:10.1115/1. 4005896.

Shaw, B. E., K. R. Milner, E. H. Field, K. Richards-Dinger, J. J. Gilchrist, J. H. Dieterich, and T. H. Jordan (2018), A physics-based earthquake simulator replicates seismic hazard statistics across California, Science Advances, 4(8), eaau0688, doi:10.1126/sciadv.aau0688.

Shi, Q., S. Barbot, S. Wei, P. Tapponnier, T. Matsuzawa, and B. Shibazaki (2020), Structural control and system-level behavior of the seismic cycle at the Nankai Trough, Earth, Planets and Space, 72(1), 27, doi:10/gm4t7p.

Shi, Z., and S. M. Day (2013), Rupture dynamics and ground motion from 3-D rough-fault simulations, Journal of Geophysical Research, [Solid Earth], 118(3), 1122-1141, doi:10.1002/ jgrb.50094.

Shibazaki, B., and Y. Iio (2003), On the physical mechanism of silent slip events along the deeper part of the seismogenic zone, Geophysical research letters, 30(9), doi:10.1029/2003GL017047.

Tal, Y., and B. H. Hager (2018), The Slip Behavior and Source Parameters for Spontaneous Slip Events on Rough Faults Subjected to Slow Tectonic Loading, Journal of Geophysical Research, [Solid Earth], 123(2), 1810-1823, doi:10.1002/2017JB014737.

Thakur, P., Y. Huang, and Y. Kaneko (2020), Effects of Low-Velocity Fault Damage Zones on Long-Term Earthquake Behaviors on Mature Strike-Slip Faults, Journal of Geophysical Research: Solid Earth, 125(8), e2020JB019,587, doi:10/gm3rfn.

Thomas, M. Y., N. Lapusta, H. Noda, and J. P. Avouac (2014), Quasi-dynamic versus fully dynamic simulations of earthquakes and aseismic slip with and without enhanced coseismic weakening, Journal of Geophysical Research, [Solid Earth], 119(3), 1986-2004, doi:10.1002/ 2013JB010615.

Tinti, E., E. Casarotti, T. Ulrich, T. Taufiqurrahman, D. Li, and A.-A. Gabriel (2021), Constraining families of dynamic models using geological, geodetic and strong ground motion data: The $\mathrm{Mw}$ 6.5, October 30th, 2016, Norcia earthquake, Italy, Earth and Planetary Science Letters, 576, 117,237, doi:10/gnzv45.

Tse, S. T., and J. R. Rice (1986), Crustal earthquake instability in relation to the depth variation of frictional slip properties, Journal of geophysical research, 91(B9), 9452, doi:10.1029/ JB091iB09p09452.

Tullis, T. E., K. Richards-Dinger, M. Barall, J. H. Dieterich, E. H. Field, E. M. Heien, L. H. Kellogg, F. F. Pollitz, J. B. Rundle, M. K. Sachs, D. L. Turcotte, S. N. Ward, and M. B. Yikilmaz (2012), Generic Earthquake Simulator, Seismological Research Letters, 83(6), 959-963, doi:10.1785/0220120093. 
Tymofyeyeva, E., Y. Fialko, J. Jiang, X. Xu, D. Sandwell, R. Bilham, T. K. Rockwell, C. Blanton, F. Burkett, A. Gontz, and S. Moafipoor (2019), Slow slip event on the southern San Andreas fault triggered by the 2017 Mw 8.2 Chiapas (Mexico) earthquake, Journal of Geophysical Research: Solid Earth, 124(9), 9956-9975, doi:10.1029/2018jb016765.

Van Dinther, Y., T. V. Gerya, L. A. Dalguer, F. Corbi, F. Funiciello, and P. M. Mai (2013), The seismic cycle at subduction thrusts: 2. Dynamic implications of geodynamic simulations validated with laboratory models, Journal of Geophysical Research, [Solid Earth], 118(4), 1502-1525, doi:10.1029/2012JB009479.

Wang, L., and S. Barbot (2020), Excitation of San Andreas tremors by thermal instabilities below the seismogenic zone, Science Advances, 6(36), eabb2057, doi:10/gm4t7q.

Wollherr, S., A.-A. Gabriel, and P. M. Mai (2019), Landers 1992 "Reloaded": Integrative Dynamic Earthquake Rupture Modeling, J. Geophys. Res. Solid Earth, 124(7), 6666-6702, doi:10/gkr74c.

Xu, J., H. Zhang, and X. Chen (2015), Rupture phase diagrams for a planar fault in 3-D full-space and half-space, Geophys. J. Int., 202(3), 2194-2206, doi:10/gkr74f.

Zhu, W., K. L. Allison, E. M. Dunham, and Y. Yang (2020), Fault valving and pore pressure evolution in simulations of earthquake sequences and aseismic slip, Nat Commun, 11(1), 4833, doi:10/gm4t7t. 